\title{
The Role of the Rural Context in the Transition to Adulthood: A Scoping Review
}

\author{
Melissa Pearman Fenton ${ }^{1}$ (D $\cdot$ Larry F. Forthun $^{1} \cdot$ Saprina Aristild ${ }^{2} \cdot$ Katherine B. Vasquez $^{1}$
}

Received: 30 March 2021 / Accepted: 11 May 2021 / Published online: 10 June 2021

(c) The Author(s), under exclusive licence to Springer Nature Switzerland AG 2021

\begin{abstract}
Rural adolescents are transitioning to adulthood in the context of growing disparities. To advance research on the social, behavioral, and contextual factors that influence rural young adult development, this study conducted a scoping review. The review sought to identify how researchers defined rural and how/which theories guided their work; how they integrated the rural context into the research design and methods; and how they used variables, concepts, and outcomes to measure rural experiences. Included articles were published between January 2009 and November 2020, included young adults ages 18-29, measured adult role achievement and/or behavioral health outcomes and reported on these outcomes for young adults, and focused on a rural sample within the United States. A systematic search of four databases resulted in 25 empirical articles for the inductive, qualitative analysis. Most studies used atheoretical approaches focusing on outcomes related to adult social roles, substance use, and mental health. Five themes emerged focused on the definitions of rural, the level of integration into the research design and method, and variables salient to the rural experience. These results reveal that future research should clearly define the rural context and better integrate the rural context into the conceptualization, design, methods, and implications of the empirical research.
\end{abstract}

Keywords Rural $\cdot$ Young adult $\cdot$ Transition to adulthood $\cdot$ Social development $\cdot$ Behavioral health

\section{Introduction}

Rural communities in the United States face increasing challenges, such as higher poverty rates, slower employment growth, aging populations, youth out migration, higher youth suicide deaths, and higher mortality rates than urban areas (Fontanella et al., 2015; United States Department of Agriculture [USDA], 2018; 2019; Cosby et al., 2019). In addition, rural areas experience disparities in access to behavioral health resources, healthcare providers, and community services (Andrilla et al., 2018; Jones et al., 2009). Rural young adults are transitioning to adulthood in the context of these growing disparities; however, their experiences

Melissa Pearman Fenton

mfenton1@ufl.edu

1 Department of Family, Youth and Community Sciences, University of Florida, 3041 McCarty D, PO BOX 110310, Gainesville, FL 32611, USA

2 Morsani College of Medicine, University of South Florida, 12901 Bruce B. Downs Blvd. MDC BOX 40, Tampa, FL 33612, USA remain understudied. To move this body of research forward and acknowledge the heterogenous factors in rural contexts, White et al. (2016) proposed contextually informed theorizing to integrate structural variables, process variables, and systemic heterogeneity in the study of human development. To identify ways that the transition to adulthood, rural context, and social and behavioral outcomes are integrated into the existing empirical literature about rural young adults, a scoping review was conducted and gaps in the literature were identified.

\section{Transition to Adulthood}

Research exploring the transition from adolescence to young adulthood has focused on the transition into adult roles generally encompassing five broad domains: work, education, romantic relationships, living independently, and family formation. This empirical research generally explores the timing of adult role transitions and the relationship to various behavioral health and social development outcomes, which may be unique in rural populations. For example, Maggs and colleagues (2012) identified four pathways among 
25-26-year-olds in a sample from the Monitoring the Future study largely defined by educational attainment, parenthood, marriage status, and living arrangements (e.g. with parents, independently, or with a partner). They found that earlier role transitions were related to decreased substance use, more economic independence, and life satisfaction in young adults; while young adults who completed a college degree, were employed fulltime, and without children, reported the highest levels of alcohol and drug use. Similar patterns have been found in research within rural communities. Carr and Kefalas (2011) identified five distinct pathways to adulthood in a sample of rural young adults from Iowa that encompassed the broader categories of Leavers, Returners, and Stayers. In their study, individuals were distinguished by educational pursuits, family formation, early work experience, and geographic relocation. Overall, the pathways identified in previous empirical studies included trajectories defined by higher education and family formation yet highlighted the heterogeneity of both transitions and outcomes experienced by young adults in nationally representative and rural contexts. Schulenberg and Schoon (2012) stressed the need for continuing to research the transition to adulthood using comparative research to gather more evidence for relationships between different role patterns and behavioral health outcomes. This research is necessary to clarify transitions and trajectories specific to rural populations where differing patterns of social roles and contextual factors may influence risk behaviors and mental health outcomes.

\section{Life Course Theory}

Several theoretical frameworks have been used to frame the research on the transition to adulthood. The most common theory to describe adult role transitions is life course theory, which describes transitions and trajectories as elements of "established pathways, their individual life courses, and developmental patterns" (Elder \& Shanahan, 2007, p. 684). Transitions are embedded within trajectories, which give transitions specific form and meaning, and describe sequences of roles and expectations of those roles over an extended period. Trajectories then form pathways, sequences of social positions in and between organizations and institutions, that structure the direction of people's lives. Of additional importance in life course theory is the focus on context, which is described as "the social embedding of individuals and typically entails study of biographical, historical, and ecological variations" (Elder et al., 2015, p. 6).

\section{Bioecological Systems Model}

Bronfenbrenner's bioecological model emphasizes how context influences young adults' social and behavioral transition to adulthood. Bronfenbrenner and Morris (2007) describe the process, or the interactions between the organism and the environment, as the core of the bioecological model. Context is represented by four ecological systems: the microsystem, mesosystem, exosystem, and macrosystem. Although proximal processes are the most influential developmental process in contributing to developmental outcomes, the geographic and cultural context that one resides in cannot be unlinked from the proximal processes. Thus, the bioecological systems model is the appropriate theoretical framework for an analysis of how the rural context influences young adult social and behavioral development.

\section{Emerging Adulthood}

Arnett (2000) proposed emerging adulthood theory as a new developmental stage characterized by the delay of adult roles and responsibilities observed in western cultures. The five features of emerging adulthood proposed by Arnett (2007) are identity explorations, instability, self-focus, feeling inbetween, and possibilities/optimism. However, critics of emerging adulthood theory point out that opportunities for young adults are shaped by structural constraints and access to resources which impacts entrance into adult roles (Côté, 2014; Hendry \& Kloep, 2007), and that this lack of attention to context in emerging adulthood theory prevents it from being widely applicable to the examination of the transition to adulthood among rural young adults.

\section{The Current Study}

A scoping review was conducted to advance research on the social, behavioral, and contextual factors that influence role transitions, risk behaviors, and mental health outcomes during the transition to adulthood among adolescents and young adults in rural communities in the U.S. A scoping review is particularly useful for summarizing and disseminating research findings, identifying gaps, and providing recommendations for further inquiry when a body of literature is large, complex, and heterogenous in nature. A scoping review was conducted to answer the following research questions: How do researchers define rural in their study samples and what theories guide their work? How do researchers integrate the rural context into the research design and method? And lastly, what variables, concepts, and outcomes are used to measure the rural experience?

\section{Methods}

The scoping methodology proposed by Arksey and O'Malley (2005) and further refined by Levac et al. (2010) and Peters et al. (2015) was used in the current study. Unlike systematic 
reviews, a scoping review does not include a formal evaluation of the methodological quality and merits of studies and aims to provide an overview of the existing literature base (Levac et al., 2010; Peters et al., 2015). To facilitate the collection of the literature, a systematic process was used to minimize bias according to PRISMA guidelines for scoping reviews (Tricco et al., 2018) (see Fig. 1).

\section{Search and Study Selection Process}

Articles were identified through searches conducted in PsycINFO, Academic Search Premier, Web of Science, and Education Resources Information Center (ERIC). The first criterion for inclusion was at least one data point from the age range included in young adulthood. Young adults were defined as individuals between the ages of 18 and 29, to encompass the age range recommended by the National Research Council (2015) (18-26) and include the emerging adult literature, which generally indicates young adults have reached adulthood by age 30 (Arnett, 2000). A second criterion was that articles measure adult role achievement and/ or behavioral health outcomes and report on these outcomes for young adults. The third criterion for inclusion was the presence of a rural sample within the United States. Rural was not defined by the research team to ensure that integration and definitions of rural contexts could be analyzed. Articles were required to be published between 2009 and 2020 to address the growing disparities in rural contexts. Only English language publications were included. A full description of search terms and exclusion criteria are outlined in Table 1. The initial search and screening were completed in August 2019. An additional search was completed in November 2020 for empirical studies published since the initial search. The resulting list of 25 articles is described in Table 2 and represents research that examined a wide variety of factors and processes related to development during the transition to adulthood in rural contexts.

\section{Data Extraction and Analysis}

Per recommendations from Arksey and O'Malley (2005) and Peters et al. (2015), a standardized data extraction form was used to collect the following information from each empirical article: citation, theoretical framework, method, variables, measures, sample characteristics, ecological system of variables, results, and discussion. The primary authors

Fig. 1 Search process

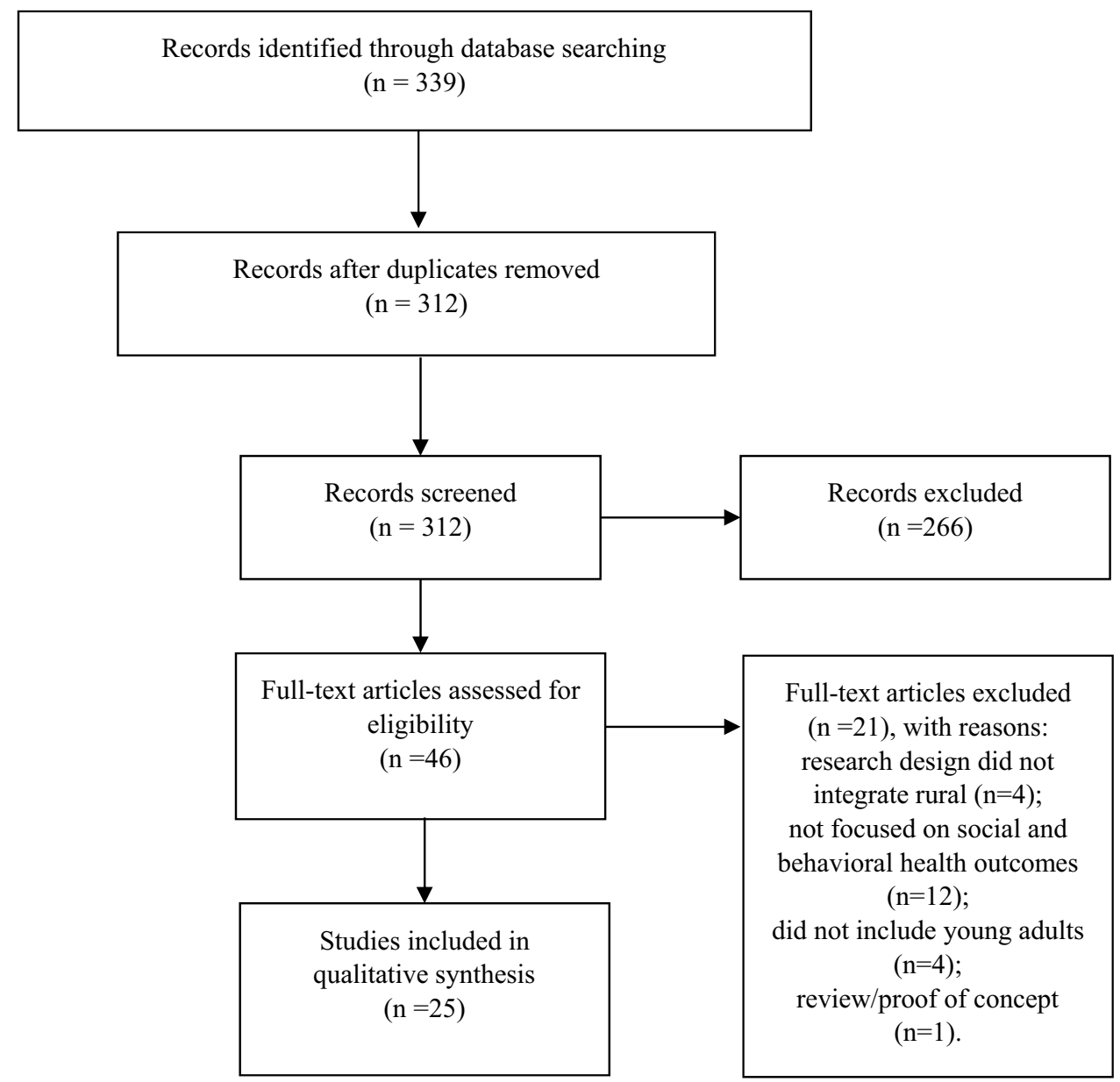


Table 1 Search inclusion and exclusion criteria

\begin{tabular}{|c|c|c|}
\hline Criteria & Inclusion & Exclusion \\
\hline Sampling search terms & $\begin{array}{l}\text { "young adult*” OR "early adult*” OR "emerging adult*” } \\
\text { OR "18-29" }\end{array}$ & $\begin{array}{l}\text { The study did not include young adults (age 18-29) in the } \\
\text { analysis }\end{array}$ \\
\hline $\begin{array}{l}\text { Social behavioral devel- } \\
\text { opment search terms }\end{array}$ & $\begin{array}{l}\text { "transition to adulthood" OR "dev* traject*" OR "role } \\
\text { traject*" }\end{array}$ & $\begin{array}{l}\text { The study was not focused on the social and behavioral } \\
\text { health outcomes of young adults }\end{array}$ \\
\hline Contextual search terms & "rural" & The research design did not intentionally integrate rurality \\
\hline Methods & $\begin{array}{l}\text { Empirical research using quantitative or qualitative } \\
\text { methods }\end{array}$ & Literature or critical reviews \\
\hline Language & English & Published in language other than English \\
\hline Publish date & $2009-2020$ & Prior to 2009 \\
\hline Publication type & Peer-reviewed journal articles & $\begin{array}{l}\text { Non-peer reviewed publications, dissertations, books, book } \\
\text { chapters, government reports }\end{array}$ \\
\hline
\end{tabular}

and a team of three graduate and undergraduate students reviewed each article and completed the data extraction forms. Following guidance for rural contextual analysis from White et al. (2016), data extraction included the identification and organization of predictor and outcome variables by ecological system (Bronfenbrenner, 1979). This required the primary author and student research assistants to review the measured variables in each study and determine through consensus its contextual position within the ecological system (i.e., is it a microsystem, mesosystem, exosystem, or macrosystem variable?). The information from this phase of analysis was used to create a descriptive summary of the included studies and for later thematic contextual analysis (see Table 2).

Guided by the research questions, the analysis was undertaken by the two primary authors using an inductive approach. Levac et al. (2010) recommended that the data analysis phase of a scoping review follow a systematic coding process. Using a three-step process, the coders reviewed the data extraction forms, including the ecological system variable analysis, and identified relevant patterns within the data as initial codes. Once initial codes were identified and refined, they were organized into categories based on similarity and continuity of content. If needed, the original research studies were consulted for clarification of coding and categorization. While useful for organizing the codes, a third step was required to organize the categories into higher-order themes. Through discussion and consensus, the authors developed a three-tier coding system composed of themes, categories (or sub-themes), and codes, which are summarized in Table 3.

\section{Results}

\section{Descriptive Summary of Studies}

Twenty-five articles were identified from the search. Table 2 presents study characteristics including method, sample size, sample characteristics, geographic location, and a brief report of major findings from each study. Of the studies reviewed, 19 were quantitative and six were qualitative. All the quantitative studies were longitudinal in nature. Three studies collected data from as early as childhood (age 9) to predict functioning into young adulthood (ages 19-30); five studies analyzed data from early adolescence (ages 10-12) to predict outcomes in young adulthood (ages 21-30); five studies focused on the changes occurring between later adolescence (16-18) and young adulthood (ages 19-25); and six studies focused solely on change in the young adulthood period (ages 18-25). Qualitative studies represented the minority of the research literature and used a variety of approaches to examine rural young adult development. Qualitative approaches included life history narrative approach, coded interviews with focus groups, a phenomenological study with in-depth interviews, narrative inquiry, and a large-scale qualitative interview study.

\section{Thematic Analysis}

Five themes emerged from the review documenting the various ways researchers defined and integrated the rural context in their research and the variables, concepts, and outcomes they explored.

\section{Theme 1: Clear Definitions of Rural were Based on Population, Proximity to Major Cities, and Economic Disadvantage}

Half of the reviewed studies (52\%) lacked a clear definition of the population size or community characteristics in their 


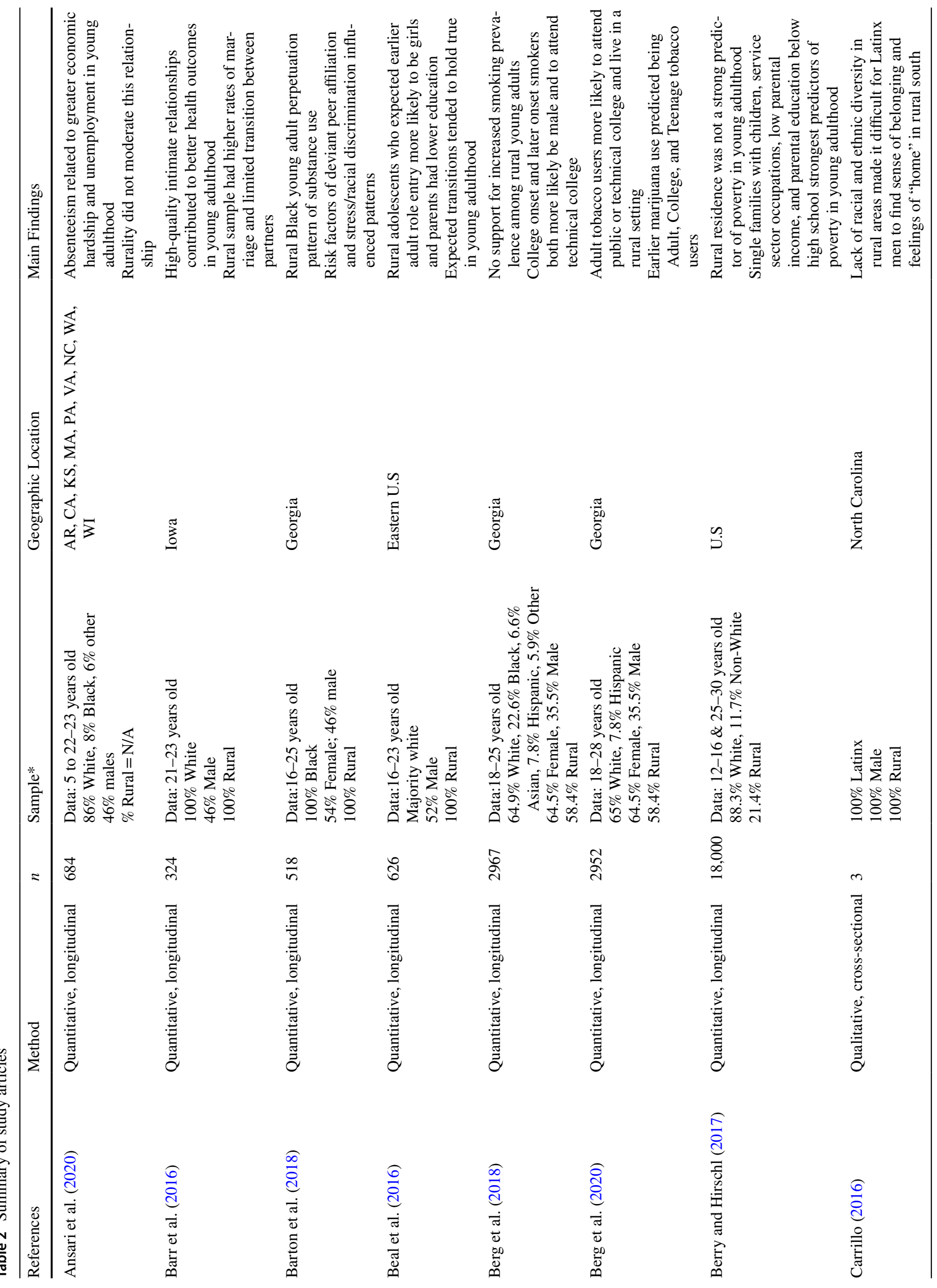




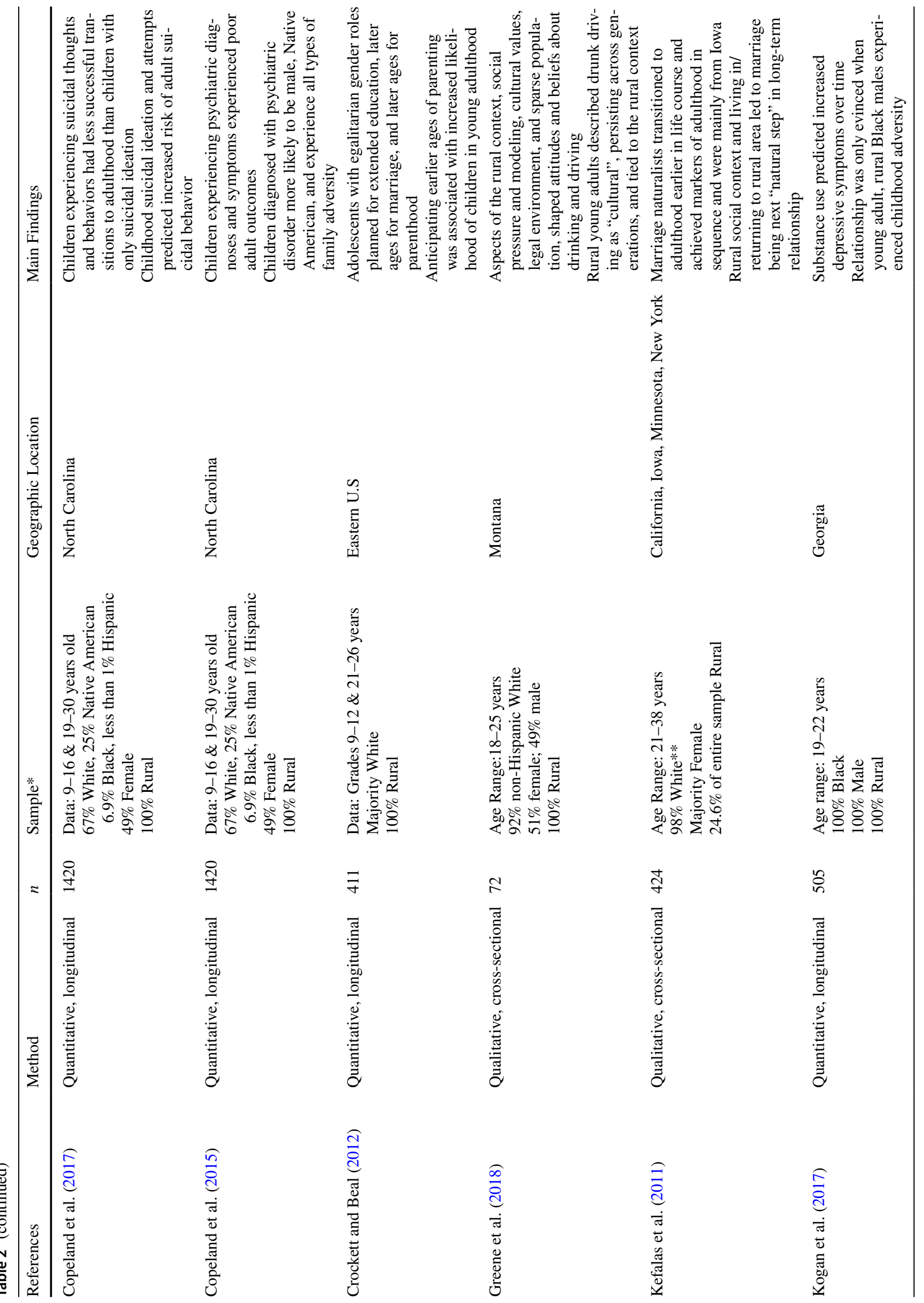




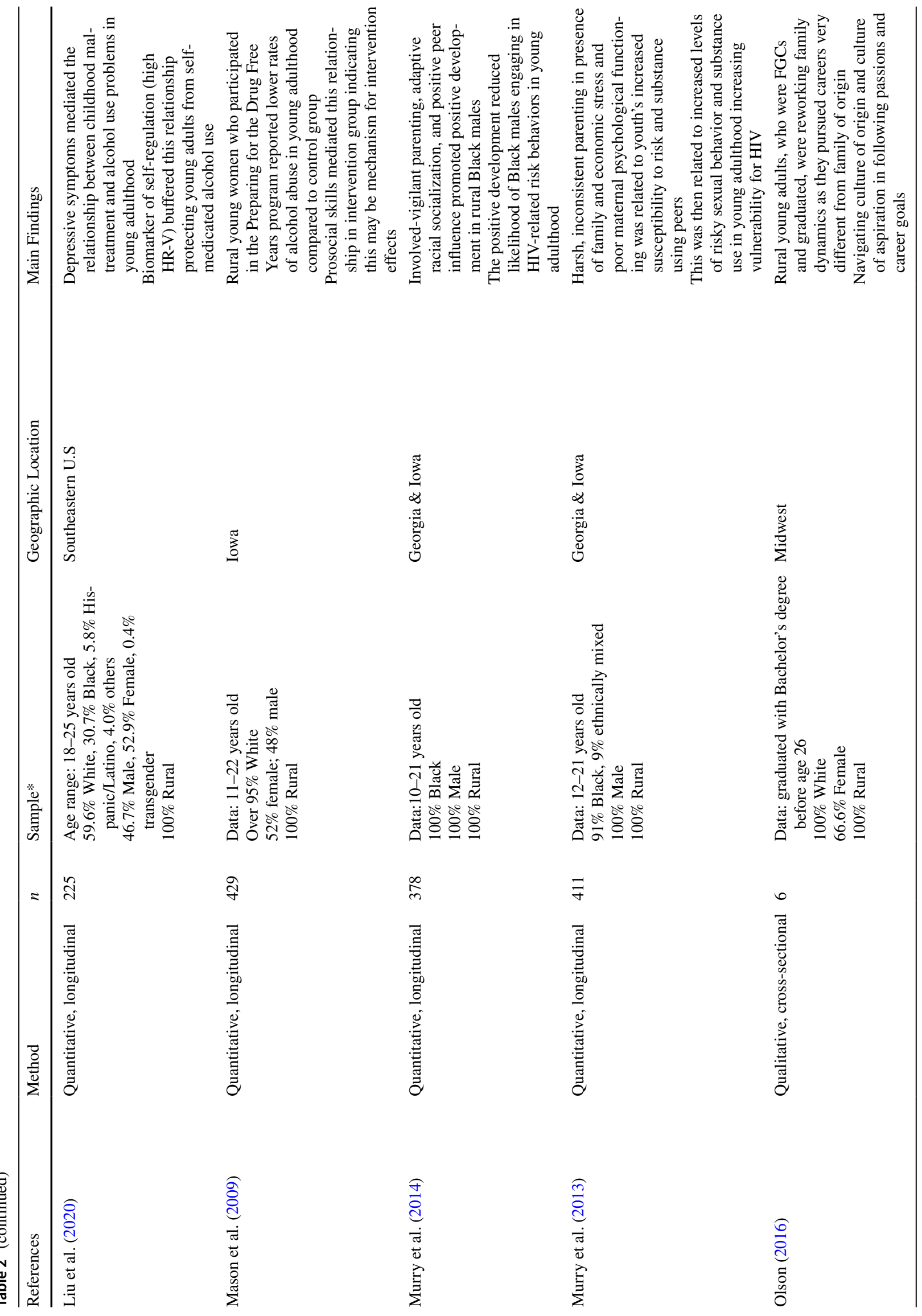




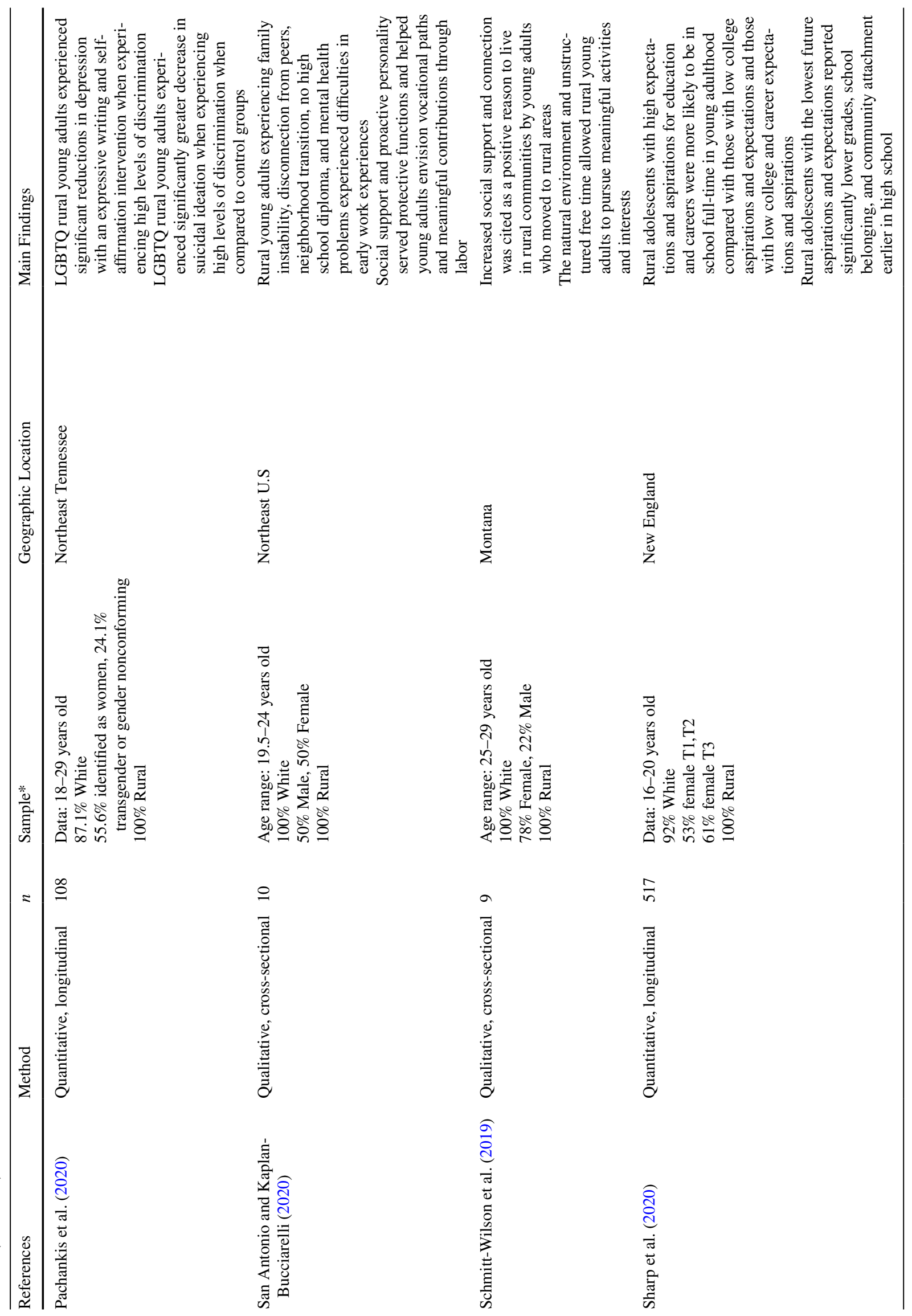




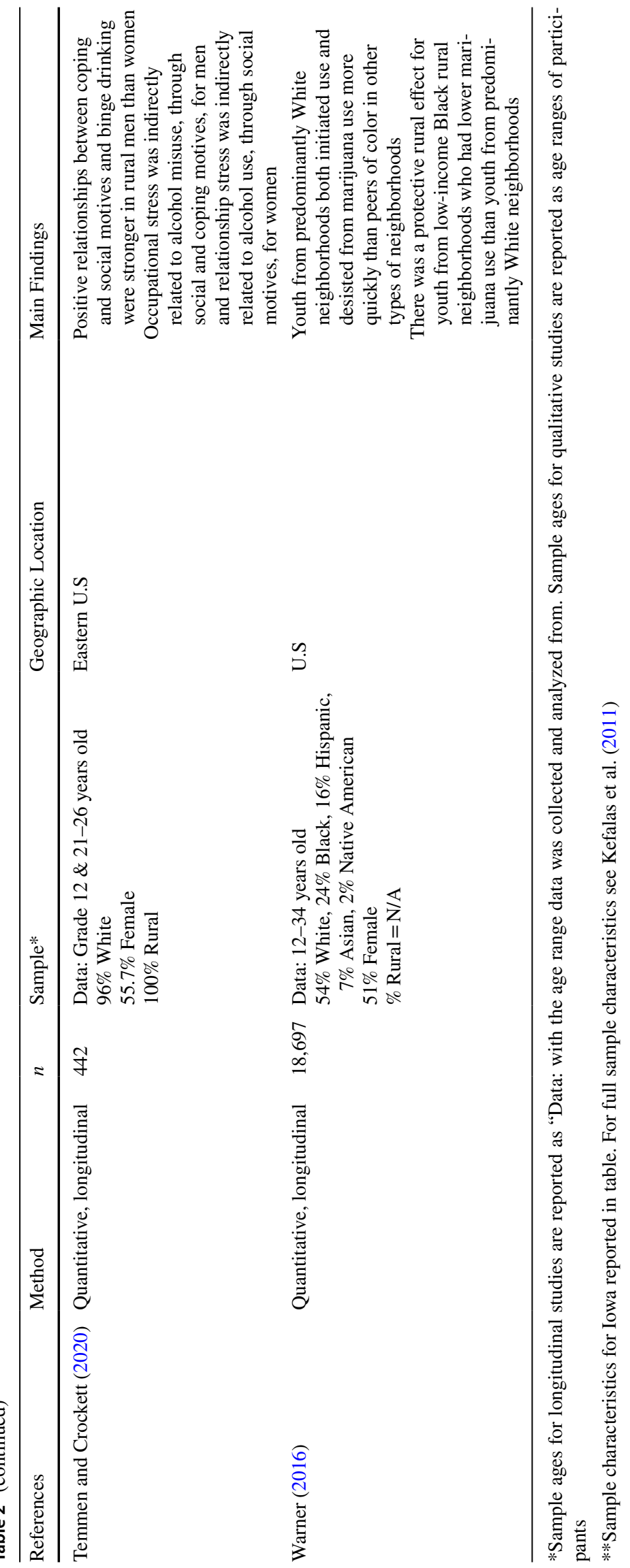




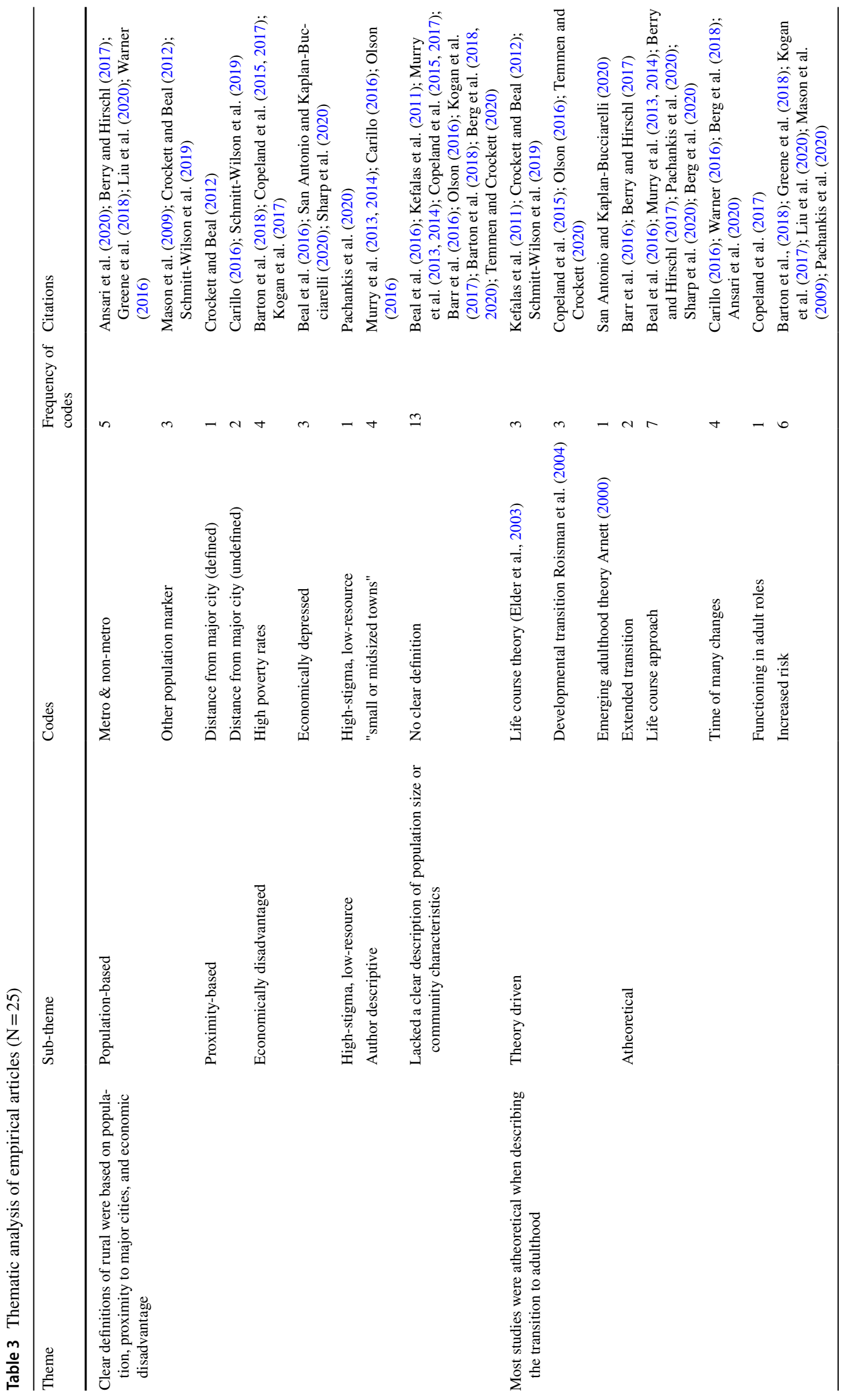




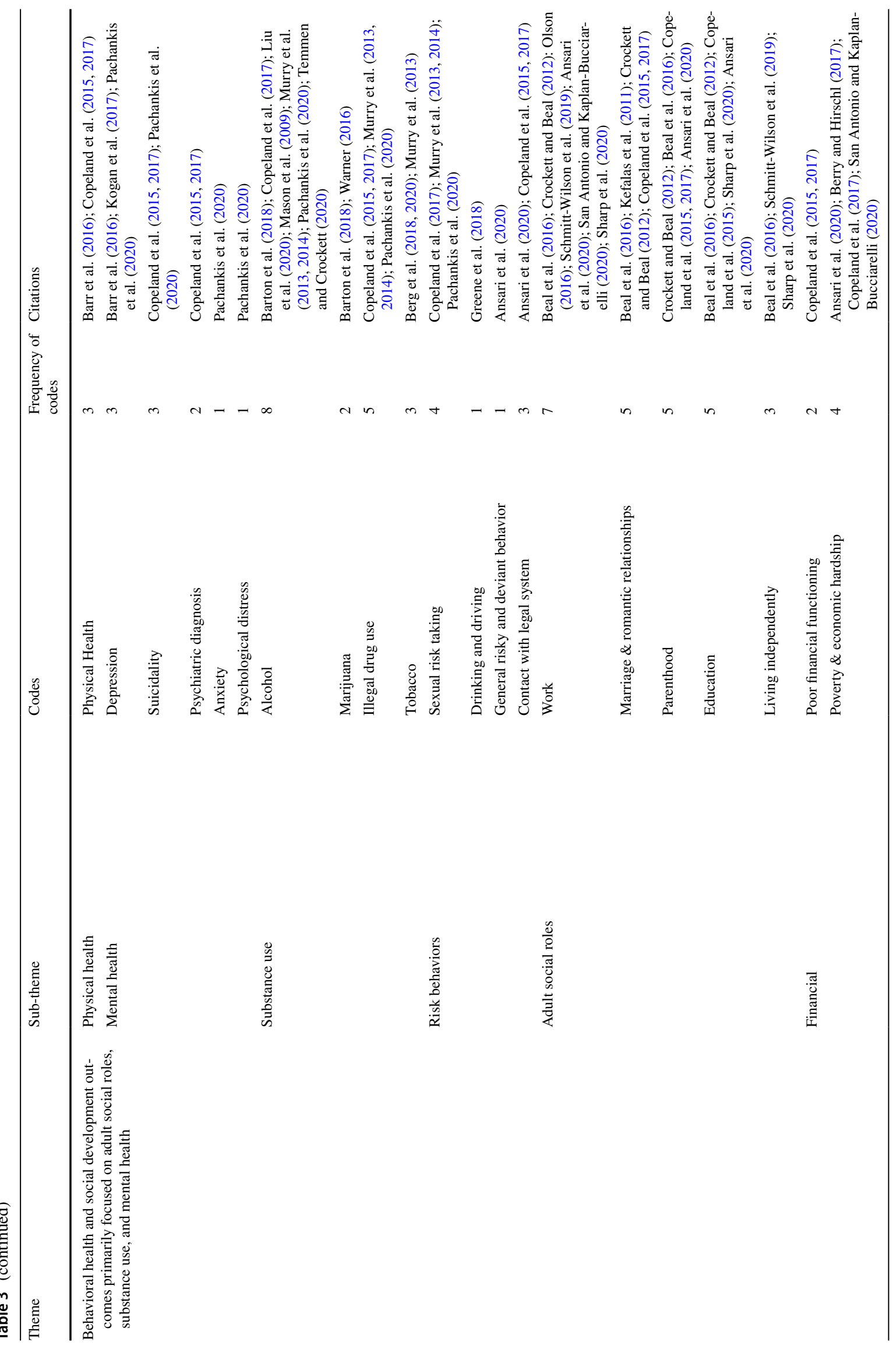




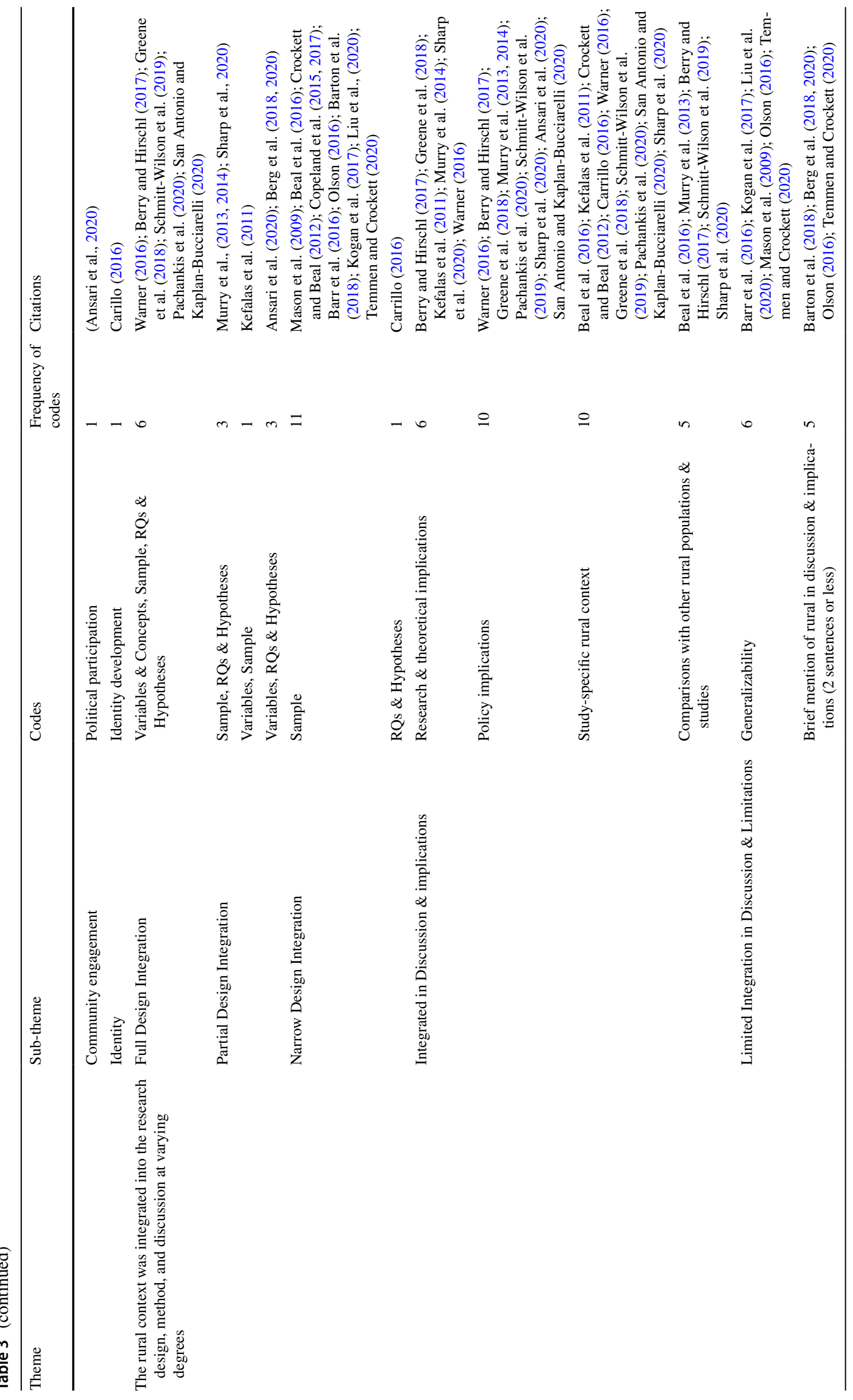




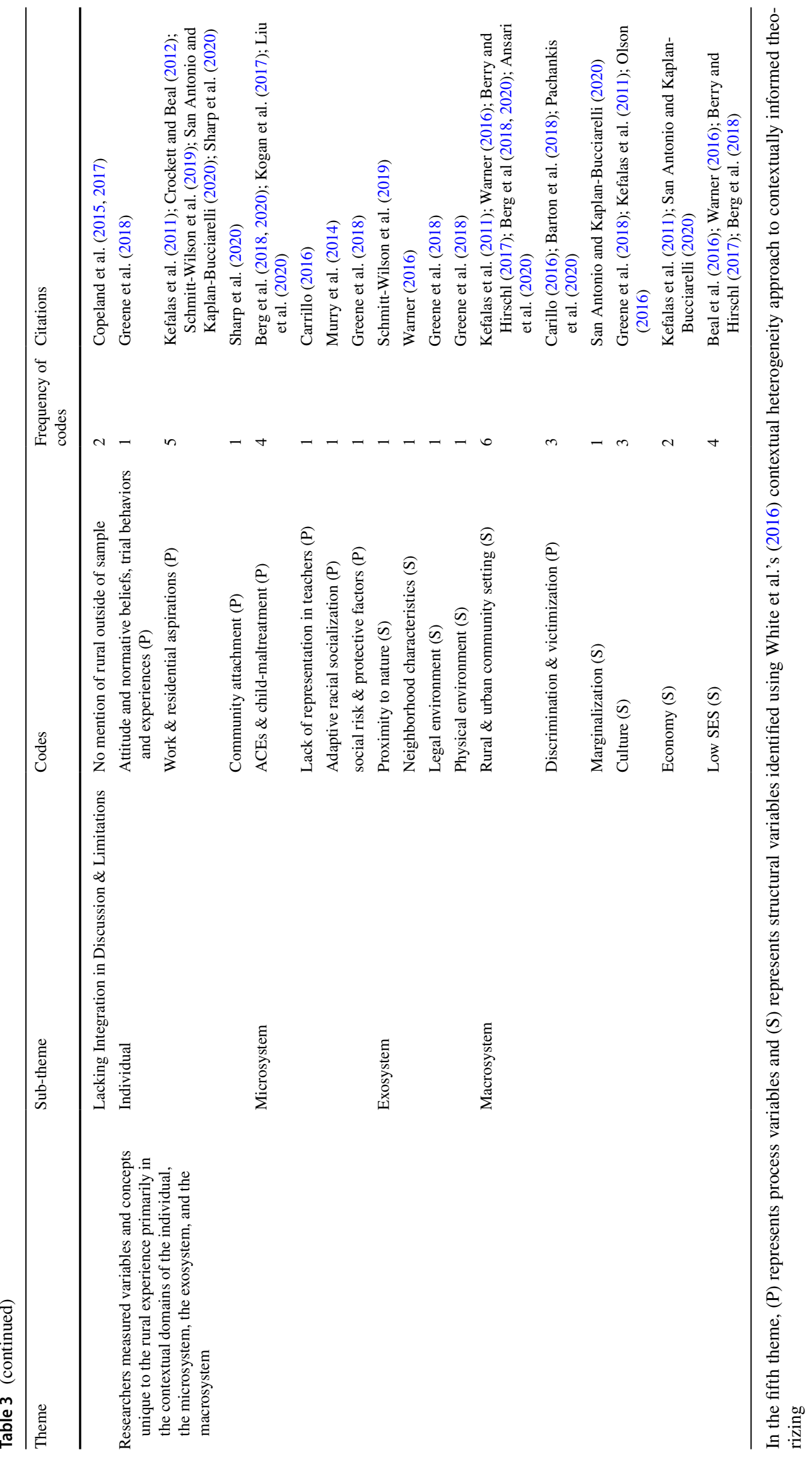


rural sample (see Table 3). These studies generally cited their sample as "rural" without further discussion or definition of what they specifically meant by "rural". Studies used population indicators, such as metro and non-metro county-level classification (U.S. Census Bureau, 2020) $(n=5)$, while others mentioned another population marker: primary town size of 3500 people (Crockett \& Beal, 2012), communities ranging from 900 to 9700 people in a Frontier Area (Schmitt-Wilson et al., 2019) and populations less than 8500 people (Mason et al., 2009). Two studies cited proximity to major cities or towns (Crockett \& Beal, 2012; Carrillo, 2016), both with a clear definition of the distance (e.g. $3 \mathrm{~h}$ from the nearest major city and $45 \mathrm{~min}$ from nearest urbanized area; Crockett \& Beal, 2012) and vague (e.g. small town outside of larger metropolitan area; Carrillo, 2016). When describing their rural sample, several studies documented high poverty rates $(n=5)$ or described the area as "economically depressed" $(n=3)$. One study described the rural area as "high-stigma, low-resource" (Pachankis et al., 2020). Kogan et al. (2017) described their rural environment as "resource poor" with a concentration of poverty that corresponds with the worst economic and health disparities by race in the U.S. Several authors also described the rural sample as hailing from "small or midsized towns" $(n=4)$.

In addition, the geographical representation of rural communities in the U.S. was limited. The majority of studies used rural samples from Georgia $(n=6)$ and Iowa $(n=5)$ (see Table 2). Three studies used samples from rural North Carolina, three studies from rural areas in the Eastern U.S., two studies from rural areas in the Northeastern U.S./New England, one study from the rural Midwest, two studies from rural Montana, one study from rural areas in the Southeastern U.S., and one from Northeastern Tennessee. Only two of the 25 studies used nationally representative samples in their research.

Authors did not justify a lack of rural definition; however, the general mention of region and categorizing the sample as rural may have been an attempt to protect participant identities. If researchers were too specific about town size within a geographic region, there could be risk of participants being identified, especially in very small communities. Additional contextual details could also be limited due to page limits and focus of the journal's articles were published in. Studies that were coded as limited or lacking rural integration (see Theme 5) tended to be vague in their definition of rural. For example, Copeland et al. (2015) did not mention the rural context in their study, except in the sample description, and did not include a clear definition of rural. However, Greene and colleagues (2018) integrated the rural context at every step in their research design and included a rich description of the rural context of their empirical study. If a researcher appeared interested in uniquely rural phenomenon or focused deliberately on a rural problem, there appeared to be more detail about the rural context.

\section{Theme 2: Most Studies were Atheoretical When Describing the Transition to Adulthood}

Of the 25 studies reviewed, seven (28\%) cited and described theory during their discussion of the transition to adulthood. Three studies integrated concepts from life course theory into their descriptions of the transitional phase of young adulthood, characterized by changing social roles and expectations within the current historical and social context (Elder $\&$ Shanahan, 2007). One study framed their investigations using emerging adulthood theory (Arnett, 2000). Three studies used a developmental transition lens that cited specific developmental tasks that young adults accomplish before transitioning to adulthood, such as developing identity, decision making skills, completing an education, and getting and maintaining a job (Roisman et al., 2004; Settersten $\&$ Ray, 2010). Of these seven studies, six were focused on social development outcomes (e.g. timing of adult social role achievement, timing and views of marriage, work outcomes, living independently in rural communities). It appears that social development outcomes may lend themselves more readily to a specific theory, especially life course theory, as there are clearer theoretical linkages.

Most studies (72\%) were atheoretical when describing the transition to adulthood, although they may have included discussions of other theories that guided their work. This may be due to the general goals of behavioral health research to identify risk and protective factors to prevent substance use and mental health problems in young adults. These studies described an extended transition, time of many changes, need to function in adult roles, and a time of increased risk in introductions to their research questions and objectives. Seven studies situated the developmental transition within the life course but did not specifically cite or use life course theory as a guiding framework.

While most studies were atheoretical, all 25 of the studies highlighted why it is important to study the transition to adulthood, as it is a sensitive period during the life course. Berg et al. (2020) described young adulthood as a "pivotal developmental period". One article asserted that "a successful transition to adulthood involves a bevy of developmental challenges" (Copeland et al., 2015, p. 893). Many articles also highlighted the elongated transition to adulthood and the need to understand how this is impacting both behavioral health outcomes and social role transitions. Contextual factors including the global restructuring of the economy, increase in educational expectations, and geography were highlighted as contributing to the elongated transition and constraining developmental opportunities for young adults. However, in the behavioral health literature these contextual 
factors were not consistently identified as being salient to the development of substance use disorders or the mental health of rural young adults. Researchers included more contextual factors in their discussion of the transition to adulthood in the social development literature. For example, Kefalas et al. (2011) described how geography plays a role in the social and personal expectations for transitions into adult social roles within the life course. Berry and Hirschl (2017) described how delays in the timing of first marriage, rise in single parent households, and global restructuring of the economy contribute to an increased risk of poverty for young adults during this transition. Akin to life course theory, context has an important role in providing and constraining opportunities for young adult development.

\section{Theme 3: Behavioral Health and Social Development Outcomes Primarily Focused on Adult Social Roles, Substance Use, and Mental Health}

An additional focus of this scoping review was on the behavioral health and social development outcomes of rural young adults in the transition to adulthood. Behavioral health outcomes were divided into four categories, substance use $(n=18)$, which was identified separately due to the number of studies that examined substance use, and physical health $(n=3)$, mental health $(n=10)$, and risk behaviors $(n=9)$. Social development outcomes were organized to represent the five broad domains of adult social roles including: work $(n=7)$, marriage and romantic relationships $(n=5)$, parenthood $(n=5)$, education $(n=5)$, and living independently $(\mathrm{n}=3)$. Additionally, poor financial functioning $(\mathrm{n}=2)$, poverty or economic hardship $(n=4)$, community engagement $(n=1)$, and identity development $(n=1)$ were included under the umbrella of social development outcomes.

\section{Behavioral Health and Social Development Outcomes Only} three studies assessed both behavioral health and social development outcomes. Copeland et al. (2015, 2017) assessed a wide variety of indicators of young adult functioning including contact with the legal system, suicidality, illegal drug use, financial functioning, marriage/romantic relationships, and parenthood, among other outcomes. Similarly, Ansari et al. (2020) assessed risk behavior and contact with the legal system in addition to parenthood, education, and poverty or economic hardship. Each study broadly focused on functioning in young adulthood and identified markers for poor functioning and early role transitions. Of note, early role transitions are not harmful in and of themselves. Early role transitions may have a protective effect for certain risk behaviors (e.g. heavy drinking, drug use, marijuana use), but a risk factor for other risk behaviors (e.g. cigarette smoking, opioid use) (Maggs et al., 2012; Green et al., 2017; San Antonio \& Kaplan-Bucciarelli, 2020). The unique combinations of adult social roles and the ages they are assumed appeared to be important factors for risk and protective effects.

Behavioral Health Outcomes Only Behavioral health outcomes are necessary to study during the transition to adulthood as substance use, risk behaviors, and mental health concerns peak around this time (Schulenberg \& Zarrett, 2006). Further, there is a disparity in access to mental health services to improve behavioral health outcomes in rural populations (Ziller \& Coburn, 2018). Liu et al. (2020) specifically highlighted the rationale for studying alcohol use problems in a rural sample was to inform prevention and intervention programs targeting rural young adults. Similarly, Murry and colleagues (2013; 2014) described the need to apply findings to address HIV/AIDS disparities among African American males in the rural south. These researchers appeared to be motivated to address health disparities in rural populations.

Social Development Outcomes Only Due to the transitional nature of young adulthood, social development outcomes tended to focus broadly on transitions to adult social roles. Longitudinal quantitative studies examined how future aspirations and expectations in adolescence were related to actual adult social role transitions, educational and work attainment, and residential aspirations in young adulthood (Beal et al., 2016; Sharp et al., 2020; Crockett \& Beal, 2012;). Four of the six qualitative studies conducted inquiries into social role transitions including the meaning of marriage for rural young people (Kefalas et al., 2011), work (Olson, 2016; San Antonio \& KaplanBucciarelli, 2020; Schmitt-Wilson et al., 2019), and living independently in rural communities (Schmitt-Wilson et al., 2019). The intersection of rural and social class appeared to constrain work and educational opportunities (San Antonio \& Kaplan-Bucciarelli, 2020; Sharp et al., 2020) while the rural context appeared to promote an earlier transition to marriage in young adulthood (Kefalas et al., 2011). Notably, only one article focused on identity development in young rural Latinx males in the context of the transition to adulthood (Carrillo, 2016), a time when personal, racial, gender, and other identities are more fully explored (Arnett, 2007).

\section{Theme 4: The Rural Context was Integrated into The Research Design, Method, and Discussion at varying Degrees}

Empirical articles were evaluated based on how well the authors integrated the rural context into the design, method, and discussion in each report (see Table 3). Sub-themes were organized into two categories, those related to design and method and those related to discussion and implications. 
Three sub-themes emerged for design and method and were defined by the level of integration in three areas: (a) studies that integrated predictor variables, concepts, covariates, and moderators that were uniquely rural or a rural variable; (b) studies that described sample selection that included the rationale for selecting the rural sample; and (c) studies that proposed research questions and hypotheses that included the rural context.

Full Design Integration Six studies achieved full design integration and specifically used rural location as a predictor for investigating behavioral health and social development outcomes. For example, the aim of a study of rural young adults' entry into poverty was to "examine whether young adults in rural areas face a heightened risk of entry into poverty" (Berry \& Hirschl, 2017, p.77) and evaluated if the residential "rural effect" persisted after controlling for contextual, family background, and individual level risk factors for poverty (c: questions). They included rural as a predictor for entering poverty in young adulthood (a: variables) and described how they identified their rural sample using Census metro and non-metro designations (b: sample).

Partial Design Integration Seven studies were identified as having partial design integration and included two of the three coding criteria (see Table 3). Most of the studies in this sub-theme either integrated rural within sample selection and research questions and hypotheses (b and c), or predictor variables and research questions and hypotheses (a and c). Only one study, Kefalas et al. (2011) integrated rural within concepts and sample selection ( $a$ and b). Kefalas and colleagues (2011) were interested in the meaning of marriage for young adults and sampled from rural Iowa to represent rural young people and included rural in their conceptualization of marriage naturalists, who tended to follow 1950s patterns of marriage and family formation. Several studies $(n=8)$ with partial integration noted poverty or areas in economic decline as a reason for selecting a rural sample. Poverty rates were higher in nonmetro areas when compared to metro areas in every year since 1959 (USDA, 2020). Behavioral health and social development outcomes are linked to socioeconomic status, so it is no surprise that rural samples are selected to study this linkage (National Center for Education Statistics, 2002; Newacheck et al., 2003; Osgood et al., 2005).

Narrow Design Integration Narrow design integration studies included only one of the criteria, most often either sample selection (b) or research questions and hypotheses (c). Eleven studies (44\%) cited rural in sample selection but did not integrate this rural context into their variables/concepts of investigation or include rural in their research questions or hypotheses. For example, Barr et al. (2016) selected a rural sample to replicate a study testing their model describing how relationship instability is associated with changes in mental and physical health; however, none of the predictor variables or research questions specifically addressed rural contexts. Uniquely, Carrillo (2016) integrated rural into the research question for the narrative life history inquiry of Latinx young men but did not include it as a concept or cite it in sample selection.

Research and Theoretical Implications The integration of rural in the discussion and implications sections of the articles was evidenced in the following ways: research and theoretical implications, policy implications, using the rural context of the study to provide potential explanations for results, and comparisons with other rural populations and/or studies. Research and theoretical implications $(n=6)$ included supporting theories of rural poverty (Berry \& Hirschl, 2017); an explanatory model of reasons for and against drinking and driving (Greene et al., 2018); geography and cultural contexts (rural) that shape orientations towards marriage for young adults (Kefalas et al., 2011); strength-based, resilience models that foster positive development among rural youth (Murry et al., 2014); different socialization processes for rural male and female adolescents that influence future aspirations and contributes to the rural brain drain (Sharp et al., 2020); and conceptualizing neighborhood risk of problem behaviors using the dimensions of race/ethnicity, class, and geography (rural) (Warner, 2016). The authors of these empirical studies discussed their findings in relation to the contributions to theories and models or providing support for further research pathways into contextual influences of behavioral health and social development outcomes.

Policy Implications Articles that cited specific policy implications $(n=10)$ ranged from macro-level changes in occupational opportunities for rural young adults to the need to consider geography in intervention and prevention programs. Prevention and intervention were the focus of many studies exploring behavioral health $(\mathrm{n}=6)$. Ansari et al. (2020) recommended reducing absenteeism in both rural and urban schools to prevent poor outcomes in young adulthood. Pachankis et al. (2020) described the potential for a highly impactful and cost-effective brief, portable intervention that could be disseminated broadly to sexual minority young adults living in high-stigma, low-resource areas. Murry and colleagues $(2013,2014)$ described how their research into risk and protective factors should be used in HIV prevention programs to address the HIV/AIDS epidemic among Black communities in the rural South. Warner (2016) called for more place-based prevention programs targeting risk behaviors in adolescents. Greene et al. (2018) recommended the risk and protective factors identified in their study be used to inform prevention programming and increase DUI enforce- 
ment to reduce drinking and driving behaviors. Several studies $(n=4)$ highlighted a need to connect rural young people to careers and/or economic opportunities such as aligning secondary education to trades (Sharp et al., 2020), provide occupational opportunities at a reputable salary and advertising these through online channels (SchmidtWilson et al., 2019), opportunities for career exploration (e.g., Makerspaces) (San Antonio \& Kaplan-Bucciarelli, 2020), and investing in local community colleges to provide educational programs to young adults from disadvantaged backgrounds (Berry \& Hirschl, 2017). The remaining studies $(n=9)$ described implications for research, theory, and policy more broadly.

Study-Specific Rural Contextual Explanations Fifteen studies used the specific rural context to explain results and/or compare their findings with other studies of rural young adults. The heterogenous nature of rural communities supports the idea that the rural-specific context may indeed influence the results of empirical studies. The economic disadvantage, loss of economic opportunities specific to that geographic location, or agrarian economy and lifestyle were described as possible contributors in six studies (Beal et al., 2016; Crockett \& Beal, 2012; Kefalas et al., 2011; Schmitt-Wilson et al., 2019; San Antonio \& Kaplan Bucciarelli, 2020; Sharp et al., 2020). Two studies highlighted the structural challenges of residing in rural communities such as disparities in mental health providers and racial or sexual identity discrimination (Carrillo, 2016; Pachankis et al., 2020). Greene et al. (2018) described the rural culture that promotes drinking and driving among young adults and Warner (2016) described a rural protective effect for lowincome, Black youth who appeared to have lower marijuana use trajectories than their white peers.

Comparisons with Other Rural Populations/Studies Five articles integrated comparisons to other rural young adult populations and studies into their discussion and implications sections. Beal et al. (2016) highlighted similarities between their sample in educational attainment, labor force participation, age of marriage, and age at parenthood and population trends for rural young people based on 1990 U.S. Census data. Murry et al. (2013) noted that their findings were consistent with previous studies of African American families living in the rural Southern U.S.

Limited Integration of Rural Eleven studies were coded as having "limited integration of rural". Six studies mentioned rural in their limitations section to caution against generalizing findings to urban samples. Five studies briefly (less than 2 sentences) mentioned the rural context in their discussion and implications section. For example, Berg and colleagues (2020) explained their finding that adult users of tobacco products were more likely to reside in rural settings due to fewer smoke-free policies and greater smoking prevalence in rural areas, structural factors that influence long-term tobacco use. In these limited integration articles, rural contexts did not appear to be central to the interpretation of findings. Temmen and Crockett (2020) cited their use of a mostly white, rural community sample as a possible explanation for the mediating effect of social motives in the linkage between normative stress and alcohol misuse during the transition to adulthood. Researchers appeared to be more focused on drawing conclusions for the effectiveness of interventions, applicability of a theory, risk and protective factors, risk trajectories, and the transition to work.

Lacking Integration of Rural Two studies lacked rural integration and mentioned rural only in reference to the sample. Copeland and colleagues $(2015 ; 2017)$ used the term "rural" only in the description of their sample. There was brief mention that the area sampled from experienced poverty and economic decline; however, it was not integrated further into the discussion or conclusions. These two studies were focused on psychopathology and adult outcomes, which may explain the lack of rural integration.

The rural context appeared to be deeply ingrained in the conceptualization and discussion of the empirical research in some articles and was treated as an afterthought in others. Varying degrees of integration in the research design and in the discussion and implications of the findings were evaluated. Overall, seven studies met the narrow design integration criteria and only two studies were lacking integration in the discussion and limitations section supporting that researchers are focusing on questions, variables, samples, and implications that are important for furthering the understanding of rural young adult development.

\section{Theme 5: Researchers Measured Variables and Concepts Unique to the Rural Experience Primarily in the Contextual Domains of the Individual, the Microsystem, the Exosystem, and the Macrosystem}

The analysis showed that the variables that were included to measure the rural environment (e.g., rural attitudes and beliefs, community attachment, etc.) were assessed at various ecological levels. Using Bronfenbrenner's (1979) ecological systems as a framework, variables were coded as representing the individual, microsystem, exosystem, and macrosystem (see Table 3). In addition, there was significant heterogeneity in the measurement of rural variables as well as the impact of the rural environment on outcomes of both quantitative and qualitative analysis. To organize these diverse characteristics, an adaptation of White et al.'s (2016) contextual heterogeneity approach to contextually informed theorizing was used. Variables were coded in three domains: 
structural variables, process variables, and systemic heterogeneity. First, variables were coded for systemic heterogeneity, which is identifying "where in the nested ecological system a relevant contextual variable belongs" (White et al., 2016, p. 282). Then, variables were coded to represent either a rural structural variable or a rural process variable. Structural variables are described as a context's key structural characteristics such as demographic, environmental, and institutional resources that are heterogenous within rural communities (White et al., 2016). These structural variables appear to primarily be represented in the selected studies within the macrosystem and exosystem. Process variables are described as an extension of Bronfenbrenner and Morris's (2007) proximal processes and evaluate the processes and interactions with other people, objects, and symbols in the developing person's environment (White et al., 2016). Process variables that appear to be unique to rural contexts included the individual level variables of community attachment, attitudes and normative beliefs, and work and residential aspirations.

Macrosystem Level The macrosystemic level was primarily characterized by structural variables like rural community setting, culture, economic realities and poverty, and marginalization but also included processes variables such as discrimination and victimization. Among the structure variables, rural community setting $(n=6)$ was included as a variable representing the macro-level concept of geography's importance to development. Rural and urban community setting was examined as a factor influencing how absenteeism impacts young adult outcomes (Ansari et al., 2020), cigarette smoking and tobacco usage (Berg et al., 2018, 2020), poverty (Berry \& Hirschl, 2017), attitudes toward marriage (Kefalas et al., 2011), and marijuana use trajectories (Warner, 2016). As noted previously, population markers for what was being considered "rural" were not always provided, which limits the information available on the variables representing the macrosystemic structure.

The rural culture was described in three studies. Participants in focus groups described the rural culture as "independence, stoicism, and a work hard, play hard attitude" (Greene et al., 2018, p. 197). While these cultural values are not solely present in rural areas or representative of all rural communities, the unique combination of these values appeared to epitomize a rural culture that encouraged risky behaviors (e.g. binge drinking, drinking and driving). Additionally, Greene et al. (2018) described social cohesion as a risk factor in rural culture due to gossip and perceptions among tight-knit community members. This may be unique to small communities where it is easy to recognize an individual's vehicles, behavior patterns, and acquaintances. The rural culture also promoted earlier marriage and a natural progression from a long-term relationship to a marital relationship for young adults in rural communities (Kefalas et al., 2011). These attitudes may be described as "old fashioned"; however, participants in this study described marriage as part of the natural order of adult roles and without other opportunities in rural communities, the next logical choice.

The rural economy and low socio-economic status were hypothesized to influence young adult development with Kefalas et al. (2011) and San Antonio and Kaplan-Bucciarelli (2020) describing a rural economy based on farming, which afforded the option of purchasing a home and establishing a family, as well as a rural economy based on low-wage jobs in service, agriculture, manufacturing, and retail industries. Poverty rates in rural areas (16.4\% in 2017) have surpassed those in urban areas (12.9\% in 2017) (USDA, 2019). While poverty is not a uniquely rural macrosystem level risk factor, it is more prevalent in rural areas, especially those more remote and rural counties (USDA, 2019). Rural areas suffer higher poverty rates and this macrolevel factor of low socio-economic status was evaluated as a risk factor in four studies including cigarette smoking (Berg et al., 2018), poverty during the transition to adulthood (Berry $\&$ Hirschl, 2017), and marijuana use trajectories (Warner, 2016). Beal and colleagues (2016) highlighted how family socio-economic status (measured as parental education) influenced expected and actual timing of young adult role transitions, highlighting how class constrains opportunities. Youth, whose parents had lower educational attainment, were more likely to anticipate early transitions into adult roles and experienced early adult role entry when compared to their peers. Lower social class is an important predictor of earlier role transitions and lower educational attainment for rural young adults. This is closely linked to the rural economy that historically experienced higher levels of unemployment and less job growth than urban areas, until the COVID-19 pandemic (Cromartie et al., 2020).

San Antonio and Kaplan-Bucciarelli (2020) described the marginalization of white rural young adults without a highschool diploma, highlighting the lack of systems to support them as they experience trauma and loss during adolescence and a lack of economic opportunities in their communities. This lack of systems, again, may be heterogenous to the region of the study (rural New England); however, rural communities in general face a lack of institutions dedicated to improving the transition for these marginalized populations (Jensen et al., 2003). As a process variable, discrimination and victimization were described in reference to Black, Latinx, and LGBTQ populations in rural areas, highlighting that they may be at greater risk for experiencing discrimination in rural communities (Carrillo, 2016; Barton et al., 2018; Pachankis et al., 2020), which may be detrimental to their well-being and development. This is not a novel finding, indeed, in a recent book edited by Crockett and Carlo 
(2016), authors review the risk and protective factors of rural, minoritized young people and their families and identify the macrosystemic process variables that predict wellbeing. Here too, a lack of systems to support rural minority young people experiencing discrimination and victimization is a concern in rural communities and creative ways will need to be employed to provide support to these marginalized young adults.

Exosystem Level Exosystemic structural level variables included proximity to nature (Schmitt-Wilson et al., 2019); neighborhood characteristics (e.g. neighborhood class, neighborhood ethnic diversity, neighborhood racial composition, street connectivity, neighborhood exposure) (Warner, 2016); and the legal and physical environment as risk factors for young adult drinking and driving (Greene et al., 2018). These variables and concepts were all cited as shaping and contributing to work and educational aspirations, residential aspirations and choices, and risk behaviors (i.e. substance abuse and drinking and driving). Warner (2016) assessed neighborhood characteristics and identified a rural protective effect, but only for Black youth living in low-income, rural neighborhoods and not white youth living in lowincome, rural neighborhoods. The physical environment also was cited as being important in young adult development. The lack of transportation options, sparse population, and knowledge of roads were highlighted as risk factors for drinking and driving, while proximity to nature was cited as a major reason for young adults migrating to rural communities (Greene et al., 2018; Schmitt-Wilson et al., 2019).

Microsystem Level At the microsystem level, process variables like Adverse Childhood Experiences (ACEs) and childmaltreatment appear to be more prevalent in rural communities (Sedlak et al., 2010; Crouch et al., 2019), which was specifically cited as a reason for selecting a rural sample to study how this impacted rural young adult development (Liu et al., 2020). Carrillo (2016) cited the lack of Latinx representation in rural teachers in primary and secondary education as a barrier to feeling at "home" in the new Latin south. Likewise, adaptive racial socialization, assessed by Murry et al. (2014), is not necessarily unique to rural families, but unique to Black families. This concept addresses caregivers' communications, behaviors, and interactions with children to facilitate adaptation, a sense of belongingness to one's race, and coping skills to deal with the realities of racism and racial discrimination in the U.S. Finally, social risk factors and protective factors for drunk driving behaviors unique to rural communities included modeling by community members, the "least drunk drive" rule, and community tragedies (i.e. young people dying as a result of drunk driving) (Greene et al., 2018).
Individual Level Finally, individual level process variables and concepts that appeared to be unique to the rural context were attitudes and normative beliefs and trial (experimental) behaviors and experiences related to drinking and driving (Greene et al., 2018); work and residential aspirations (Kefalas et al., 2011; Crockett \& Beal, 2012; San Antonio \& Kaplan-Bucciarelli, 2020; Schmitt-Wilson et al., 2019; Sharp et al., 2020); and community attachment (Sharp et al., 2020). Work and residential aspirations were identified as individual level factors as aspiring to live in a rural community is uniquely rural, as are certain occupational aspirations (e.g. farming). These specific work and residential aspirations are especially integrated into the rural context and structured by economic opportunities in the area. Community attachment refers to the emotional investment in place (Humon, 1992). However, in somewhat counterintuitive findings, Sharp et al. (2020) found that rural adolescents who reported high aspirations for their future education and career success reported the highest levels of community attachment but were the least likely to report wanting to remain in their home county. This is also contrary to findings that youth with higher attachment to their communities in high school were more likely to live in a rural area in young adulthood (Johnson et al., 2005; Looker \& Naylor, 2009).

By using White et al.'s (2016) contextual heterogeneity approach to contextually informed theorizing to analyze and categorize variables within the research literature, the current study highlighted approaches to evaluating both structural and process variables that represented various systems within the ecosystem of rural young people. Notably, most of the structural variables operated at the exosystem and macrosystem levels. Process variables were represented in the microsystem, individual, and macrosystem ecological systems. The identification of both structural and process variables was necessary to identify both gaps for potential study and ideas for new ways to integrate these variables into future studies to increase the understanding of how the rural context influences young adult development.

\section{Discussion}

Both empirical and anecdotal evidence suggest that adolescents living in rural communities experience several unique challenges in the transition to adulthood. However, much of the contemporary research examining the behavioral and social development of young adults has failed to consider these unique individual and contextual characteristics of rural life. The purpose of this review was to examine the contemporary literature on the transition to adulthood to better understand how researchers define rural in their study samples, identify the theories that guided their work, and 
integrate rural variables, concepts, and outcomes into the research design, methods, and discussion. Following guidelines proposed by PRISMA for scoping reviews (Tricco et al., 2018), 25 published empirical articles, 19 quantitative and 6 qualitative, were identified and coded for analysis. Five themes emerged from the textual analysis and highlight both the strengths and weaknesses of contemporary approaches to studying the transition to adulthood.

\section{Rural Context and Geographic Representation}

The analysis found that half of the studies included in this review (52\%) lacked a clear definition of the population size or community characteristics of the rural sample. While journal page limits, intent, and focus may limit the ability of researchers to present a full contextual description of the rural community participants were recruited from, contextual information is important. Rural communities are heterogenous with differences in community strengths and deficits to be accounted for in the interpretation of results. Providing additional contextual descriptions can help communities identify commonalities and generalize findings to their own young adult populations, improving the reach of policies and programs designed to help young adults. Notable exceptions include Pachankis et al. (2020) who provided a rich description of the "high-stigma, low-resource" rural area where they tested their intervention. The thorough description was intentional to aid in the replication and dissemination of similar online, brief writing interventions to improve mental health and health risk-behaviors for LGBTQ young adults. Greene et al. (2018) also provided significant contextual details about the rural area in Montana where they conducted their research on risk and protective factors for drinking and driving. These contextual descriptions are necessary to extend findings to other communities and identify differences in risk and protective factors that may influence the efficacy of evidence-based interventions.

In addition, there are geographic gaps in research in states and regions in the U.S. with rural populations (see Table 2). Notably, the Northwest and Southwest were not represented in this scoping review. The Midwest was mainly represented by one state, Iowa, and the Southeast was represented mainly by populations in the state of Georgia. Not all rural communities are alike and it is important to extend research on rural communities to other geographic locations that include racial, ethnic, resource, and cultural diversity. For example, The American Communities Project (2020) evaluated counties across the U.S. and identified 15 different types of communities that represent the diversity of rural, urban, and suburban communities in the U.S. Researchers could use these county designations, or other demographic indicators, to identify rural communities that are underrepresented in the literature to focus new research on young adult transitions and development. Namely, rural counties identified as Hispanic Centers, Evangelical Hubs, Aging Farmlands, African American South, and Native American Lands (American Communities Project, 2020). To prevent further marginalization of populations in these rural communities, researchers should partner with local community stakeholders and members of the community to design studies and interventions to best meet the needs of these populations.

Nationally representative datasets, such as Monitoring the Future and The National Longitudinal Study of Adolescent to Adult Health, offer opportunities to examine rural young adult transitions across geographic regions in the U.S. (Furstenberg, 2016). Although there are limitations (e.g. age of datasets, measurement, attrition) these datasets could be used to better understand rural young adult transitions in a variety of rural contexts. An exciting new study, Adolescent Brain Cognitive Development (ABCD), may offer opportunities in the future to examine biological and behavioral development into young adulthood (ABCD, 2020). Through research that expands geographic representation, along with clear descriptions and definitions of the rural community, future research can overcome these current limitations, making the results more generalizable and accessible to programs and policies designed to help young adults in diverse rural contexts.

\section{Developmental and Life Course Theoretical Integration}

Life Course Theory was the most common theoretical framework used to describe the transition to adulthood in the studies identified in this scoping review. This theory is uniquely suited to explore the influence of context on the transition to adulthood. However, completing the transition to adulthood also includes emerging developmental tasks like success in work and romantic relationships (Roisman et al., 2004). Thus, a focus on the adult social roles in the domains of work, education, parenthood, romantic relationships, and living independently should include both a developmental (i.e. how do behaviors and competence in the salient developmental tasks of adolescence predict emergence into adult roles) and contextual focus.

However, the importance of including both behavioral health and social development outcomes in studies should not be overlooked. Further integration of social development and behavioral health outcomes would help to better understand how these emerging developmental tasks and social roles are related to the risk behaviors and mental health of young adults. Some studies have accomplished this to a degree. Maggs et al. (2012) found that single young adults who were working fulltime and living with their parents reported the lowest levels of global life satisfaction and the highest cigarette smoking prevalence when compared to the 
other patterns of adult social roles. Young adults without children who worked full time, had high levels of achieved education, and were both single and in committed relationships reported the highest levels of alcohol, marijuana, other drug use, with married young adults with children reporting the lowest use of these substances. Six patterns of adult social roles were assessed for their relationship to what the researchers termed as "illegal acts" which measured how often in the last six months the respondent "damaged property; had a physical fight; drove when drunk; used marijuana; used other drugs; did something else illegal" (Osgood et al., 2005, p. 349). Slow starters, those reporting the fewest adult role transitions and from lower social classes, reported the highest rate of illegal behaviors. Certainly, the increased responsibilities of child rearing may explain the disparate use of substances and illegal behavior between patterns of adult social roles; however, additional research is needed to understand how these developmental transition patterns are related to more comprehensive pictures of behavioral health, especially in rural young adult populations. Therefore, researchers should integrate developmental theories that consider the contextual and cultural factors influencing the transition, combined with developmental tasks that include the emergence into culturally relevant adult social roles and functioning in young adulthood.

\section{Achieving Full Integration}

Analysis of the design and method of the selected empirical research studies suggests that the rural context could be fully integrated in studies by: (a) integrating predictor variables, concepts, covariates, and moderators that are uniquely rural; (b) describing sample selection that includes the rationale for selecting the rural sample; and (c) proposing research questions and hypotheses that include the rural context. Those studying rural populations are encouraged to use White et al.'s (2016) proposed strategy for contextually informed theorizing to achieve all markers of full integration. By theorizing how structural and process variables and concepts salient to the rural context relate to young adult development, researchers can provide a clear description and rationale for the rural sample and integrate these variables and concepts into research questions/hypotheses. Researchers should aim to integrate rural specific variables and concepts present at all levels of the ecological system, while achieving full integration in sample selection, research questions and hypotheses, and discussion and implications. Examples of specific variables/concepts and recommendations for integrating those variables/concepts are highlighted in the following paragraphs. These recommendations assume that inherent within the process of contextually informed theorizing, salient rural structural and process variables will be identified and described, more detailed sample descriptions will emerge, and contextually informed research questions will develop.

\section{Rural Structural Variables}

In several studies included in this scoping review, rural culture was highlighted as a structural variable that influenced risk behaviors. Similarly, researchers interested in integrating cultural variables could use qualitative methods to identify, from the populations themselves, what the rural ethos is in their context of interest. To be contextually specific, socio-economic status and poverty should also include structural variables unique to that rural community, such as informal education and nonmonetary means of meeting daily needs that do not conform to the traditional ways of measuring this variable (White et al., 2016). To accurately describe the economic status of participants and operationalize this structural variable, care should be given to what resources and deficits are present in the rural context. Murry et al. (2014) and Greene et al. (2018) provided structural explanations and support for the process variables included in their study, grounding them in the rural context. Other studies could integrate region and community-level structural variables such as reporting of child maltreatment incidences or ACEs data from their state and percentages of teachers of color in rural schools. Even if they could not be included as specific variables, framing the research study using this structural information would help extend findings to rural contexts with similar geosocial structures. Certainly, Warner's (2016) findings highlight that a protective effect for one demographic is not the same for another demographic. Thus, researchers should identify the exosystem level variable(s) important to their research question and seek to integrate them into the study. As previously argued, this attention to contextual details will aid in applying findings to other communities who identify similar structural variables.

\section{Rural Process Variables}

At the individual level, identification of new contextually appropriate process variables is needed to establish their relationship to development in the rural context. Notably missing from the selected studies was exploring how a sense of place influences rural young adult development. Community attachment is part of this concept, but it is more complex. Sense of place is how people feel about and relate to the environment they live and grow up in and is represented by three constructs: place attachment, place dependence, and place identity (Humon, 1992; Jorgensen \& Stedman, 2001). San Antonio (2016) found how salient a sense of place was to rural young adult development. "Participants spoke about expanding their experience with diversity and having a sense of place that was connected to being in a community with 
other people and, for some, having access to natural settings for solace and comfort" (San Antonio, 2016, p. 255). Future research should integrate the constructs related to sense of place, as these are likely heterogenous among minoritized and majority populations in rural communities. In addition, future studies should explore process variables that are observable in their rural context of interest. For example, mixed age peer groups in small, rural Midwest communities.

\section{Rural Mesosystem Variables}

There was a lack of variables at the mesosystem level in all studies, not just those more specific to the rural context. Mesosystem level factors are more difficult to identify, as they describe the interactions between microsystem level influences. In a study of ecological factors that influence adolescent alcohol misuse, Ennett et al. (2008) found that the interactions between family, school, and friends can both buffer against substance abuse or amplify the effects of family, school, and peer group risk factors. These findings required complicated statistical analyses (i.e. multilevel modeling) and data sources including longitudinal data from adolescents, longitudinal data from participating adolescents' parents, social network analysis of peers, and connecting this data to U.S. Census tracts and block groups. The level of complexity required to analyze mesosystem level risk and protective factors may explain why there was a lack of these factors in the reviewed studies.

\section{Rural Research, Practice, and Policy}

Likewise, there were missed opportunities to integrate discussions of how rural findings can be used to inform future research, practice, and policy. For example, there are disparities in access to health interventions in rural communities (Hartley, 2004; Centers for Disease Control, 2019). The specific recommendations for policy changes could provide clear actionable suggestions to rural communities looking to address substance use, mental health, and economic and health access disparities for rural young people. By addressing the growing health and economic disparities between rural and urban communities, researchers should be making a case for how their results inform policy, prevention, and research on rural outcomes moving forward. For instance, San Antonio and Kaplan-Bucciarelli (2020) recommend the use of narrative career counseling and life design dialogue to provide support for disconnected low-income young adults. However, their recommendation of counselors, career development specialists, and mentors necessary to help these young people does not call for additional funding programs or training in these methods for rural practitioners. The authors generally describe partnerships between schools and community organizations with examples of
Trauma Sensitive Schools and Makerspaces as opportunities to support young people and provide meaningful career explorations. Researchers describing actionable steps and highlighting ideas would give rural communities a place to start when trying to improve outcomes for rural young adults and revitalize rural communities.

\section{Prevention and Intervention}

Similarly, prevention and intervention research should document the structural and process variables that influence rural, marginalized young adults' development. Gottfredson and colleagues (2015) provided updated standards for prevention science research including effectiveness and scaling up. Within their recommendations, clear language outlining the range of application for the intervention as well as anticipated limitations to application with different populations, settings, times, and outcomes is recommended for highquality evidence-based programs. As this review has documented, there are rural contextual factors that influence risk behaviors in adolescents and young adults. Thus, prevention and intervention research should continue to document both structural and process variables salient to intervention and prevention outcomes. For example, Pachankis et al. (2020) clearly documented the high-stigma, low-resource rural context that their brief, portable writing interventions were tested in. The researchers recommended continuing to integrate local community input, guiding language and situational prompts, to ensure that internet-based writing interventions remain applicable to sexual minority populations they are designed to help. These clear descriptions of structural (few LGBTQ-specific formal support resources) and process (discrimination and victimization) variables and recommendations for implementation in other communities provide an excellent example for future research.

Only six of 25 studies were identified as reaching full integration while 13 of the 25 studies were limited or lacking integration of rural in the discussion and implications sections. Although the focus of each of these studies may not be on the unique ways the rural context shapes development of young adults, increasing the number of studies that are fully integrated in both study design and discussions will only benefit this population and help reduce disparities within rural communities.

\section{Strengths and Limitations}

There are several strengths and limitations to this scoping review. First, the review was not meant to be exhaustive, but a scope of the recent general literature about the transition to adulthood for rural young adults. Bias in selecting articles for the full review was reduced using clear exclusion and inclusion criteria; however, relevant studies may be missed. 
More targeted systematic reviews on specific behavioral health or social development outcomes would improve conclusions about how rural young adults are faring in these areas. The scoping review is limited by the choice of search terms to define the transition to adulthood. The authors hoped to include both social and developmental outcomes with the inclusion of developmental and role trajectories; however, relevant literature could be excluded that did not use these terms. A strength of this scoping review is the focus on the empirical results that were peer-reviewed and validated. However, findings and conclusions may be limited by the inclusion of only peer-reviewed sources. "Gray literature" which includes unpublished dissertations and reports could color the findings and recommendations generated by this scoping review. The findings in "gray literature", although not peer reviewed, may have explored variables representing the rural context and provided additional details about the rural communities they studied. This information would be useful for further evaluating geographic representation, theoretical approaches, outcomes, integration of rural, and systems-levels of variables. This scoping review focused on the U.S. and insights could be gained by exploring how rural young adult development is impacted by the rural context internationally. Additionally, scoping reviews are not meant to assess the quality of the empirical evidence. More targeted systematic reviews that focus on specific risk and protective factors unique to rural contexts, timing of adult social roles in young adulthood, and interventions for behavioral health outcomes would inform the field of the quality of relevant research in these areas.

\section{Conclusion}

Rural young people are transitioning to adulthood amid constrained economies and opportunities and the research into their development should be expanded to identify how this context is influencing their long-term outcomes. The results of this scoping review have highlighted several challenges for researchers who seek to better understand the transition to adulthood among young adults in rural contexts. The recommendations contained in this review advocate for more theory driven research that seeks to fully integrate what it means to be rural. Future research should incorporate the rural context in all stages of the research process and provide actionable policy implications to improve outcomes. Through outreach efforts, policy briefs, and collaborations with other experts, researchers can influence policymakers to consider the rural context in decision making. This means clearly defining the rural context; using a theoretical framework, such as life course theory, that defines the transition to adulthood; integrating behavioral health and social development outcomes; and identifying and integrating structural and process variables at multiple ecological system levels that represent unique aspects of rural communities. Only then will researchers be able to build a foundation of research that recognizes the diverse experience of rural young adults from across the U.S. and can translate that knowledge into actionable policy, prevention, and intervention activities.

Acknowledgements We thank Sofia Mora, an undergraduate research assistant at the University of Florida, for assisting with the analysis.

Authors contribution MF conceived of the study, designed the study, conducted the analysis, and drafted the manuscript; LF participated in the design and analysis and helped to draft the manuscript; SA participated in the analysis; and KV participated in the analysis. All authors read and approved the final manuscript.

\section{Declarations}

Conflict of interest The authors report no conflict of interest.

\section{References}

\section{*Denotes article included in scoping review.}

ABCD Study. (2020). About the study. Retrieved from the ABCD Study website https://abcdstudy.org/about/

American Communities Project. (2020). The American communities. Retrieved from the American Communities Project website https://www.americancommunities.org/\#map

Andrilla, C. H., Patterson, D. G., Garberson, L. A., Coulthard, C., \& Larson, E. H. (2018). Geographic variation in the supply of selected behavioral health providers. American Journal of Preventative Medicine, 54(6S3), S199-S207. https://doi.org/10.1016/j. ampre.2018.01.004

*Ansari, A., Hofkens, T. L., \& Pianta, R. C. (2020). Absenteeism in the first decade of education forecasts civic engagement and educational and socioeconomic prospects in young adulthood. Journal of Youth and Adolescence, 49(9), 1835-1848. https://doi.org/10. 1007/s10964-020-01272-4

Arksey, H., \& O’Malley, L. (2005). Scoping studies: Towards a methodological framework. International Journal of Social Research Methodology: Theory and Practice, 8(1), 19-32. https://doi.org/ 10.1080/1364557032000119616

Arnett, J. J. (2000). Emerging adulthood: A theory of development from the late teens through the twenties. American Psychologist, 55(5), 469-480. https://doi.org/10.1037/0003-066X.55.5.469

Arnett, J. J. (2007). Emerging adulthood: What is it, and what is it good for? Child Development Perspectives, 1(2), 68-73. https://doi.org/ 10.1111/j.1750-8606.2007.00016.x

*Barr, A. B., Sutton, T. E., Simons, L. G., Wickrama, K. A. S., \& Lorenz, F. O. (2016). Romantic relationship transitions and changes in health among rural, White young adults. Journal of Family Psychology, 30(7), 832-842. https://doi.org/10.1037/fam0000207

*Barton, A. W., Brody, G. H., Zapolski, T. C. B., Goings, T. C., Kogan, S. M., Windle, M., \& Yu, T. Y. (2018). Trajectory classes of cannabis use and heavy drinking among rural African American adolescents: multi-level predictors of class membership. Addiction, 113(8), 1439-1449. https://doi.org/10.1111/add.14200 
*Beal, S. J., Crockett, L. J., \& Peugh, J. (2016). Adolescents' changing future expectations predict the timing of adult role transitions. Developmental Psychology, 52(10), 1606-1618. https://doi.org/ 10.1037/dev0000189

*Berg, C. J., Haardörfer, R., Vu, M., Getachew, B., Lloyd, S. A., Lanier, A., \& Windle, M. (2018). Cigarette use trajectories in young adults: Analyses of predictors across system levels. Drug \& Alcohol Dependence, 188, 281-287. https://doi.org/10.1016/j. drugalcdep.2018.03.055

*Berg, C. J., Haardörfer, R., Lanier, A., Childs, D., Foster, B., Getachew, B., \& Windle, M. (2020). Tobacco use trajectories in young adults: Analyses of predictors across systems levels. Nicotine \& Tobacco Research, 22(11), 2075-2084. https://doi.org/10. 1093/ntr/ntaa048

*Berry, P. G., \& Hirschl, T. A. (2017). Non-metro versus metro poverty in the transition to adulthood in the United States:(1980-2009). Journal of Rural Studies, 54, 76-84. https://doi.org/10.1016/j.jrurs tud.2017.04.011

Bronfenbrenner, U. (1979). The ecology of human development: Experiments by nature and design. Harvard University Press.

Bronfenbrenner, U., \& Morris, P. A. (2007). The bioecological model of human development. In R.M. Lerner \& W. Damon (Eds.) Handbook of child psychology volume 1: Theoretical models of human development (6th ed., pp. 793-828). Wiley.

Carr, P. J., \& Kefalas, M. J. (2011). Straight from the heartland: Coming of age in Ellis, Iowa. In M. C. Waters, P. J. Carr, \& M. J. Kefalas (Eds.), Coming of age in America: The transition to adulthood in the twenty-first century (pp. 28-58). University of California Press.

*Carrillo, J. F. (2016). Searching for "home" in Dixie: Identity and education in the New Latin@ South. Educational Studies, 52(1), 20-37. https://doi.org/10.1080/00131946.2015.1120208

Centers for Disease Control. (2019, July 1). Rural health. https://www. cdc.gov/chronicdisease/resources/publications/factsheets/ruralhealth.htm

*Copeland, W. E., Goldston, D. B., \& Costello, E. J. (2017). Adult associations of childhood suicidal thoughts and behaviors: A prospective, longitudinal analysis. Journal of the American Academy of Child \& Adolescent Psychiatry. https://doi.org/10.1016/j.jaac. 2017.08.015

Copeland, W. E., Wolke, D., Shanahan, L., \& Costello, E. J. (2015). Adult functional outcomes of common childhood psychiatric problems: A prospective, longitudinal study. JAMA Psychiatry, 72(9), 892-899. https://doi.org/10.1001/jamapsychiatry.2015. 0730

Cosby, A. G., Maya McDoom-Echebiri, M., James, W., Khandekar, H., Brown, W., \& Hanna, H. L. (2019). Growth and persistence of place-based mortality in the United States: The rural mortality penalty. American Journal of Public Health, 109(1), 155-162. https://doi.org/10.2105/AJPH.2018.304787

Côté, J. E. (2014). The dangerous myth of emerging adulthood: An evidence-based critique of a flawed developmental theory. Applied Developmental Science, 18(4), 177-188. https://doi.org/10.1080/ 10888691.2014.954451

*Crockett, L. J., \& Beal, S. J. (2012). The life course in the making: Gender and the development of adolescents' expected timing of adult role transitions. Developmental Psychology, 48(6), 17271738. https://doi.org/10.1037/a0027538

Crocket, L. J. \& Carlo, G. (Eds.) (2016). Rural ethnic minority youth and families in the United States: Theory, research, and applications. Springer, Cham. https://doi.org/10.1007/978-3-319-20976-0

Cromartie, J., Dobis, E. A., Krumel, Jr., T. P., McGranahan, D., \& Pender, J. (2020). Rural America at a glance, 2020 edition (Economic Information Bulletin 221). United States Department of Agriculture Economic Research Service. https://www.ers.usda. gov/webdocs/publications/100089/eib-221.pdf?v=9990.5
Crouch, E., Probst, J. C., Radcliff, E., Bennett, K. J., \& McKinney, S. H. (2019). Prevalence of adverse childhood experiences (ACEs) among US children. Child Abuse \& Neglect, 92(April), 209-218. https://doi.org/10.1016/j.chiabu.2019.04.010

Elder G.H., Johnson M.K., Crosnoe R. (2003) The emergence and development of Life Course Theory. In: Mortimer J.T., Shanahan M.J. (eds) Handbook of the Life Course (pp. 3-19). Springer. https://doi.org/10.1007/978-0-306-48247-2_1

Elder, G. H., \& Shanahan, M. J. (2007). The life course and human development. In R. M. Lerner \& W. Damon, W (Eds.). Handbook of child psychology: Volume 1. Theoretical models of human development (6th ed.) (pp. 665-715). Wiley.

Elder, G. H., Shanahan, M. J., \& Jennings, J. A. (2015). Human development in time and place. In M. H. Bornstein \& T. Leventhal (Eds.) Handbook of child psychology and developmental science: Volume 4. Ecological settings and processes ( $\left.7^{\text {th }} \mathrm{ed}\right)(\mathrm{pp} .6-54)$. Wiley.

Ennett, S. T., Foshee, V. A., Bauman, K. E., Hussong, A., Cai, L., Luz, H., \& Durant, R. (2008). The social ecology of adolescent alcohol misuse. Child Development, 79(6), 1777-1791. https://doi.org/10. $1111 / j .1467-8624.2008 .01225 . x$

Fontanella, C. A., Hiance-Steelesmith, D. L., Phillips, G. S., Bridge, J. A., Lester, N., Sweeney, H. A., \& Campo, J. V. (2015). Widening rural-urban disparities in youth suicides, United States, 1996-2010. JAMA Pediatrics, 169(5), 466-473. https://doi.org/ 10.1001/jamapediatrics.2014.3561

Furstenberg, F. (2016). Social class and development in early adulthood: Some unsettled issues. Emerging Adulthood, 4(4), 236-238. https://doi.org/10.1177/2167696815625142

Gottfredson, D. C., Cook, T. D., Gardner, F. E. M., Gorman-Smith, D., Howe, G. W., Sandler, I. N., \& Zafft, K. M. (2015). Standards of evidence for efficacy, effectiveness, and scale-up research in prevention science: Next generation. Prevention Science. https:// doi.org/10.1007/s11121-015-0555-x

Green, M. J., Leyland, A. H., Sweeting, H., \& Benzeval, M. (2017). Causal effects of transitions to adult roles on early adult smoking and drinking: Evidence from three cohorts. Social Science \& Medicine, 187, 193-202. https://doi.org/10.1016/j.socscimed. 2017.06.018

*Greene, K. M., Murphy, S. T., \& Rossheim, M. E. (2018). Context and culture: Reasons young adults drink and drive in rural America. Accident Analysis and Prevention, 121(2018), 194-201. https:// doi.org/10.1016/j.aap.2018.09.008

Hartley, D. (2004). Rural health disparities, population health, and rural culture. American Journal of Public Health, 94(10), 1675-1678. https://doi.org/10.2105/AJPH.94.10.1675

Hendry, L. B., \& Kloep, M. (2007). Conceptualizing emerging adulthood: Inspecting the emperor's new clothes? Child Development Perspectives, 1(2), 74-79. https://doi.org/10.1111/j.1750-8606. 2007.00017.x

Humon, D. M. (1992). Community attachment: Local sentiment and sense of place. In I. Altman \& S. M. Low (Eds.), Place attachment (pp. 253-278). Plenum Press.

Jensen, L., McLaughlin, D. K., \& Slack, T. (2003). Rural poverty: The persisting challenge. In D. L. Brown \& L. E. Swanson (Eds.), Challenges for rural America in the twenty-first century (pp. 118-131). Penn State University Press.

Jorgensen, B. S., \& Stedman, R. C. (2001). Sense of place as an attitude: Lakeshore owners attitudes toward their properties. Journal of Environmental Psychology, 21, 233-248.

Johnson, M. K., Elder, G. H., Jr., \& Stern, M. (2005). Attachments to family and community and the young adult transition of rural youth. Journal of Research on Adolescence, 15(1), 99-125. https://doi.org/10.1111/j.1532-7795.2005.00088.x

Jones, C. A., Parker, T. S., Ahearn, M., Mishra, A. K., \& Variyam, J. N. (2009). Health status and health care access of farm and rural 
populations (Economic Information Bulletin Number 57). United States Department of Agriculture, Economic Research Services. https://www.ers.usda.gov/webdocs/publications/44424/9371_ eib57_1_.pdf

*Kefalas, M. J., Furstenberg, F. F., Carr, P. J., \& Napolitano, L. (2011). "Marriage is more than being together": The meaning of marriage for young adults. Journal of Family Issues, 32(7), 845-875. https://doi.org/10.1177/0192513X10397277

*Kogan, S. M., Cho, J., Oshri, A., \& MacKillop, J. (2017). The influence of substance use on depressive symptoms among young adult black men: The sensitizing effect of early adversity. American Journal on Addictions, 26(4), 400-406. https://doi.org/10.1111/ ajad. 12555

Levac, D., Colquhoun, H., \& O'Brien, K. K. (2010). Scoping studies: Advancing the methodology. Implementation Science, 5(69), 1-9. https://doi.org/10.1186/1748-5908-5-69

*Liu, S., Oshri, A., \& Duprey, E. B. (2020). Heart rate variability reactivity moderates the indirect link between child maltreatment and young adult alcohol use problems via depressive symptoms. American Journal on Addictions, 29(2), 141-150. https://doi.org/ 10.1111/ajad.13004

Looker, E. D., \& Naylor, T. D. (2009). 'At risk' of being rural? The experience of rural youth in a risk society. Journal of Rural and Community Development, 4(2), 39-64.

*Mason, W. A., Kosterman, R., Haggerty, K. P., Hawkins, D. J., Redmond, C., Spoth, R. L., \& Shin, C. (2009). Gender moderation and social developmental mediation of the effect of a family-focused substance use preventive intervention on young adult alcohol abuse. Addictive Behaviors, 34(2009), 599-605. https://doi.org/ 10.1016/j.addbeh.2009.03.032

Maggs, J. L., Patrick, M. E., \& Schulenberg, J. (2012). Social role patterning in early adulthood in the USA: adolescent predictors and concurrent wellbeing across four distinct configurations. Longitudinal and Life Course Studies, 3(2), 190-210. https://doi.org/ 10.14301/llcs.v3i2.183

*Murry, V. M., Simons, R. L., Simons, L. G., \& Gibbons, F. X. (2013). Contributions of family environment and parenting processes to sexual risk and substance use of rural African American Males: A 4-year longitudinal analysis. American Journal of Orthopsychiatry, 83(2), 299-309. https://doi.org/10.1111/ajop.12035

*Murry, V. M., Berkel, C., Simons, R. L., Simons, L. G., \& Gibbons, F. X. (2014). A twelve-year longitudinal analysis of positive youth development among rural African American males. Journal of Research on Adolescence, 24(3), 512-525. https://doi.org/10. 1111/jora.12129

National Center for Education Statistics (NCES). (2002). Education Longitudinal Study of 2002. http://nces.ed.gov/surveys/els2002/ bibliography.asp

National Research Council. (2015) Investing in the health and wellbeing of young adults. Washington, DC: The National Academies Press. https://doi.org/10.17226/18869

Newacheck, P. W., Hung, Y. Y., Park, M. J., Brindis, C. D., \& Irwin, C. E. (2003). Disparities in adolescent health and health care: Does socioeconomic status matter? Health Services Research, 38, 1235-1252. https://doi.org/10.1111/1475-6773.00174

*Olson, J. S. (2016). Chasing a passion: First-generation college graduates at work. Education \& Training, 58(4), 358-371.http://doi. org/https://doi.org/10.1108/ET-03-2015-0023

Osgood, D. W., Ruth, G., Eccles, J., Jacobs, J. E., \& Barber, B. L. (2005). Six paths to adulthood: Fast starters, parents without careers, educated partners, educated singles, working singles, and slow starters. In R. A. Settenstein, F. F. Furstenberg, \& R. G. Rumbaut (Eds.), On the frontier of adulthood: Theory, research, and public policy (pp. 320-335). University of Chicago Press.

*Pachankis, J. E., Williams, S. L., Behari, K., Job, S., McConocha, E. M., \& Chaudoir, S. R. (2020). Brief online interventions for
LGBTQ young adult mental and behavioral health: A randomized controlled trial in a high-stigma, low-resource context. Journal of Consulting and Clinical Psychology, 88(5), 429-444. https://doi. org/10.1037/ccp0000497

Peters, M. D. J., Godfrey, C. M., Khalil, H., McInerney, P., Parker, D., \& Soares, C. B. (2015). Guidance for conducting systematic scoping reviews. International Journal of Evidence-Based Healthcare, 13(3), 141-146. https://doi.org/10.1097/XEB.0000000000000050

Roisman, G. I., Masten, A. S., Coatsworth, J. D., \& Tellegen, A. (2004). Salient and emerging developmental tasks in the transition to adulthood. Child Development, 75(1), 123-133. https://doi. org/10.1111/j.1467-8624.2004.00658.x

San Antonio, D. M. (2016). The complex decision-making processes of rural emerging adults: Counseling beyond dualism. Peabody Journal of Education, 91(2), 246-269. https://doi.org/10.1080/ 0161956X.2016.1151749

*San Antonio, D. M., \& Kaplan-Bucciarelli, J. (2020). Possibility and constraint in the lives of young rural workers in the United States: The psychology of working and post-trauma vocational trajectories. Emerging Adulthood. https://doi.org/10.1177/21676 96820957812

*Schmitt-Wilson, S., Hanson, M. R., \& Vaterlaus, J. M. (2019). Young, educated and choosing rural: An exploration of the factors related to young adults choosing to move to remote rural communities. The Journal of Rural and Community Development, 14(4), 94-113. Retrieved from www.jrcd.ca

Schulenberg, J. E., \& Zarrett, N. R. (2006). Mental health during emerging adulthood: Continuity and discontinuity in courses, causes, and functions. In. J. Arnett \& J. Tanner (Eds.), Emerging adults in America: Coming of age in the $21^{\text {st }}$ century (pp.135172). American Psychological Association.

Schulenberg, J., \& Schoon, I. (2012). The transition to adulthood across time and space: Overview of special section. Longitudinal and Life Course Studies, 3(2), 164-172. https://doi.org/10.14301/llcs. v3i2.194

Sedlak, A.J., Mettenburg, J., Basena, M., Petta, I., McPherson, K., Greene, A., and Li, S. (2010). Fourth National Incidence Study of Child Abuse and Neglect (NIS-4): Report to Congress. Washington, DC: U.S. Department of Health and Human Services, Administration for Children and Families. Retrieved from https://www. acf.hhs.gov/sites/default/files/documents/opre/nis4_report_congr ess_full_pdf_jan2010.pdf

Settersten, R. and Ray, B.E. (2010). Not quite adults: Why 20-somethings are choosing a slower path to adulthood, and why it's good for everyone. Bantam Books Trade Paperbacks.

*Sharp, E. H., Seaman, J., Tucker, C. J., Van Gundy, K. T., \& Rebellon, C. J. (2020). Adolescents' future aspirations and expectations in the context of a shifting rural economy. Journal of Youth and Adolescence, 49(2), 534-548. https://doi.org/10.1007/ s10964-019-01152-6

*Temmen, C. D., \& Crockett, L. J. (2020). Relations of stress and drinking motives to young adult alcohol misuse: Variations by gender. Journal of Youth and Adolescence, 49(4), 907-920. https://doi.org/10.1007/s10964-019-01144-6

Tricco, A. C., Lillie, E., Zarin, W., O’Brien, K. K., Colquhoun, H., Levac, D., \& Straus, S. E. (2018). PRISMA extension for scoping reviews (PRISMA-ScR): Checklist and explanation. Annals of Internal Medicine, 169(7), 467-473. https://doi.org/10.7326/ M18-0850

U.S. Census Bureau. (2020, February 24). Urban and rural. Retrieved from the U.S. Census Bureau website https://www.census.gov/ programs-surveys/geography/guidance/geo-areas/urban-rural.html

United States Department of Agriculture (USDA). (2018). Rural America at a glance, 2018 ed. (Economic Information Bulletin No. 200). Retrieved from USDA Economic Research Service website 
https://www.ers.usda.gov/webdocs/publications/90556/eib-200. pdf? $\mathrm{v}=5899.2$

USDA. (2019). Rural America at a glance, 2019 ed. (Economic Information Bulletin No. 212). https://www.ers.usda.gov/webdocs/ publications/95341/eib-212.pdf? $\mathrm{v}=3322$

USDA. (2020). Poverty rates by metro/nonmetro residence, 1959-2018. https://www.ers.usda.gov/data-products/chart-gallery/gallery/ chart-detail/?chartId $=56288$

*Warner, T. D. (2016). Up in smoke: Neighborhood contexts of marijuana use from adolescence through young adulthood. Journal of Youth and Adolescence, 45(1), 35-53. https://doi.org/10.1007/ s10964-015-0370-5

White, R.M.B., Burleson, E., \& Knight, G.P. (2016) Future prospects for studying ethnic and racial minority youths and families in diverse rural and nonrural contexts. In: Crockett L., Carlo G. (eds) Rural ethnic minority youth and families in the United States (pp. 267-286). Springer, Cham. https://doi.org/10.1007/978-3-31920976-0_15

Ziller, E., \& Coburn, A. (2018, August 1). Health equity challenges in rural America. Human Rights, 43(3). https://www.americanbar. org/groups/crsj/publications/human_rights_magazine_home/thestate-of-healthcare-in-the-united-states/health-equity-challengesin-rural-america/
Publisher's Note Springer Nature remains neutral with regard to jurisdictional claims in published maps and institutional affiliations.

Melissa Pearman Fenton is a doctoral student at the University of Florida. Her major research interests include rural adolescent and young adult development with specific interest in substance use and mental health outcomes.

Larry F. Forthun is an Associate Professor at the University of Florida. His major research interests include the analysis of problematic behavior and mental health in adolescents and emerging adults, and the promotion of health, well-being, and resilience.

Saprina Aristild is a master's student at the University of South Florida. Her major research interests include health disparities, maternal/fetal health, and youth development.

Katherine B. Vasquez is a master's student at the University of Florida. Her major research interests include the transition to adulthood with a specific interest in underrepresented and marginalized student groups. 